\title{
GAIT RECOVERY IN HEALTHY SUBJECTS: PERTURBATIONS TO THE KNEE MOTION WITH A SMART KNEE BRACE
}

\author{
by \\ Mehmet Temel
}

A thesis submitted to the Faculty of the University of Delaware in partial fulfillment of the requirements for the degree of Master of Science in Mechanical Engineering

Fall 2010

(c) 2010 Mehmet Temel

All Rights Reserved 


\title{
GAIT RECOVERY IN HEALTHY SUBJECTS: PERTURBATIONS TO THE KNEE MOTION WITH A SMART KNEE BRACE
}

\author{
by
}

Mehmet Temel

Approved:

Sunil K. Agrawal, Ph.D.

Professor in charge of thesis on behalf of the Advisory Committee

Approved:

Anette M. Karlsson, Ph.D.

Chair of the Department of Mechanical Engineering

Approved:

Michael J. Chajes, Ph.D.

Dean of the College of Engineering

Approved:

Charles G. Riordan, Ph.D.

Vice Provost for Graduate and Professional Education 


\section{ACKNOWLEDGEMENTS}

I would like to thank to Dr. Sunil K. Agrawal for giving me the opportunity to work on the Smart Knee Brace project. I thank him for his advice, guidance and support in all aspects of this research. His enthusiasm and motivation for research has been inspiring for me.

I thank Dr. Katherine S. Rudolph for helping me expand my knowledge into the world of Physical Therapy. I would like to express my appreciation for her guidance, support and patience during the process.

I thank Dr. Herbert Tanner as a committee member for taking time to review my thesis and provide feedback.

I would like to thank Dr. Seok Hun Kim for his support and additional advice for the most part of my research. I would like to thank fellow students Deepak and Josh for their help. I would also like to thank Vivek, Ji-Chul, Zaeem, Melissa and Justin for their support and friendship.

I would like to express the deepest thanks to my family. Especially, to my brother for his endless support and motivation during hard times and being the greatest stress relief available during this process. 


\section{TABLE OF CONTENTS}

LIST OF FIGURES . . . . . . . . . . . . . . . . . . . . vi

LIST OF TABLES . . . . . . . . . . . . . . . . . . xii

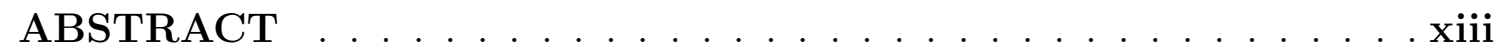

\section{Chapter}

1 INTRODUCTION . . . . . . . . . . . . . . . . . . 1

1.1 The Effects of Fall . . . . . . . . . . . . . . . . . . . . 1

1.2 Current Training Methods . . . . . . . . . . . . . . . . . 2

1.3 Smart Knee Brace Concept . . . . . . . . . . . . . . . . . . . . . . 2

1.4 Summary and Thesis Outline ............... 3

2 SMART KNEE BRACE . . . . . . . . . . . . . . . . . . . . 4

3 HARDWARE ADAPTATIONS AND THE CONTROL

ALGORITHM ..................... 7

3.1 The Hardware . . . . . . . . . . . . . . . . . . . . . . . . . . . 8

3.2 The Software . . . . . . . . . . . . . . . . . 10

3.2.1 Built-in Time Delay within the System . . . . . . . . . . . 13

3.2.1.1 Time Delay in Response to Pressure Sensor Data . . 13

3.2.1.2 Time Delay in Response to Magnetic Encoder Data . 16

3.2.2 Control Algorithm Based On Pressure Sensor Data . . . . . . 17

3.2.3 Control Algorithm Based On Pressure Sensor and Magnetic Encoder Data ................. 23

3.2.3.1 Flexion Lock in Early Swing Phase . . . . . . . . . . 24

3.2.3.2 Extension Lock in Late Swing Phase . . . . . . . . . 28 
3.2.3.3 Flexion Lock in Stance Phase . . . . . . . . . . . . . 31

3.2.3.4 Extension Lock in Stance Phase . . . . . . . . . . . 32

4 GAIT CHARACTERIZATION . . . . . . . . . . . . . 35

4.1 Dynamic Model of Human Walking During Swing Phase . . . . . . 35

4.1 .1 Equations of Motion . . . . . . . . . . . . . . 37

4.2 Human Neuro-muscular Response . . . . . . . . . . . . . . . . 40

4.2 .1 Independent Joint Control . . . . . . . . . . . . . . . . . . 41

4.2 .2 Coordinated Joint Control . . . . . . . . . . . . . . . . 45

4.2.3 Independent Joint Control with Time Shift . . . . . . . . . . 47

4.2.4 Coordinated Joint Control with Time Shift . . . . . . . . . . . 49

4.2 .5 Experimental Results . . . . . . . . . . . . . . . . 49

4.2.5.1 Data Collection . . . . . . . . . . . . . 51

4.2 .5 .2 Discussion . . . . . . . . . . . . . . . . 52

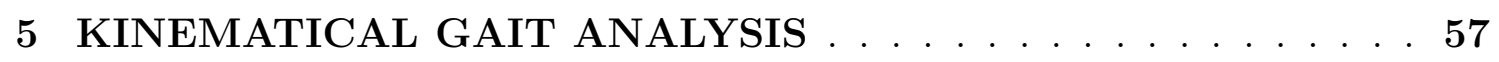

5.1 Single Lock . . . . . . . . . . . . . . . . . . . . . . . 57

5.2 Continuous Lock . . . . . . . . . . . . . . . . . 61

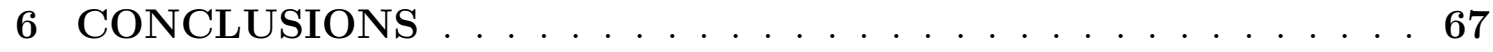

6.1 Future Work . . . . . . . . . . . . . . . . . . . . . 68

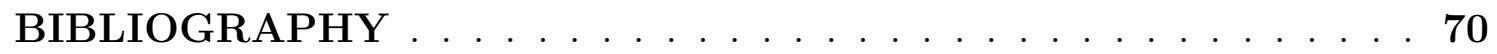




\section{LIST OF FIGURES}

2.1 The locking mechanism. Each lock is powered by a solenoid that engages and disengages a lock pawl into a gear plate. The locks function independent of each other. . . . . . . . . . . . . 5

2.2 The components and computer interface of SKB. Right knee joint angle data and pressure from four sets of sensors underneath the foot are sent by SKB. The brace receives the details of the locking condition such as lock angle, lock duration and number of engagements. . . . . . . . . . . . . . . . . 6

3.1 Right knee joint angle data is collected via the brace (blue) and motion capture system (red) while the subject is standing still on the treadmill. The difference between two lines is no more than $0.5^{\circ}$ and consistent throughout the experiment. . . . . . . . . . 10

3.2 Peak joint angles for the right (blue) and left (red) leg are shown. Before hardware improvements, (a) hip angles and (b) knee angles differ from each other approximately by $10^{\circ}$. Hardware improvements have a significant influence on (c) hip and (d) knee data, which also indicates subject's comfort with the brace. . . . . 12

3.3 Heel pressure sensor data (blue) and flexion lock status (red) are shown. Threshold value of the heel pressure sensor and the status of the flexion lock are scaled to the same constant value. The time delay within the system seems to be $40 \mathrm{~ms}$ when the sampling rate of the Target Computer is $50 \mathrm{~Hz}$. . . . . . . . . . . . . . . . . 14

3.4 Heel pressure sensor data (blue) and flexion lock status (red) are shown. The time delay within the system seems to be $30 \mathrm{~ms}$ when the sampling rate of the Target Computer is $100 \mathrm{~Hz}$. . . . . . . . 15 
3.5 Heel pressure sensor data (blue) and flexion lock status (red) are shown. The time delay within the system seems to be $15 \mathrm{~ms}$ when the sampling rate of the Target Computer is $1200 \mathrm{~Hz}$. . . . . . . . 16

3.6 Right knee angle (dashed), pressure sensor set 2 (blue) and pressure sensor set 3 (red) are shown. It takes $60 \mathrm{~ms}$ for the flexion lock to get engaged after the magnetic encoder reading reaches the preset angle value. . . . . . . . . . . . . . . . . . 17

3.7 Right knee angle (dashed), pressure sensor set 2 (blue) and set 3 (red) are shown. It takes $60 \mathrm{~ms}$ for the extension lock to get engaged after the magnetic encoder reading reaches the preset angle value. . 18

3.8 Right knee angle (dashed), pressure sensor set 1 (green), set 2 (black), set 3 (red) and set 4 (blue) are shown on the baseline gait pattern of a subject. The baseline pattern is analyzed to determine the convenient points in the gait cycle at which knee locks could be activated. . . . . . . . . . . . . . . .

3.9 Engagement of flexion lock $10 \mathrm{~ms}$ after toe-off. Pressure Sensor Set 1 (blue) data is used for heel-strike detection while Pressure Sensor Set 4 (red) is used for toe-off detection. Magnetic encoder data (dashed) shows right knee angle. . . . . . . . . . . . . . . 20

3.10 Engagement of flexion lock $0 \mathrm{~ms}$ after toe-off. Pressure Sensor Set 1 (blue) data is used for heel-strike detection while Pressure Sensor Set 4 (red) is used for toe-off detection. Magnetic encoder data (dashed) shows right knee angle. . . . . . . . . . . . . . 2

3.11 Engagement of flexion lock $15 \mathrm{~ms}$ after toe-off. Pressure Sensor Set 1 (blue) data is used for heel-strike detection while Pressure Sensor Set 4 (red) is used for toe-off detection. Magnetic encoder data (dashed) shows right knee angle. . . . . . . . . . . . . . . 22

3.12 Locking strategies during one stride (heel-strike to the next consecutive heel-strike). Flexion locks can be engaged during Loading Response and Early Swing Phase, Extension Locks can engage during Midstance and Late Swing Phase at prescribed angles. 24

3.13 A subject in the early swing phase. . . . . . . . . . 24 
3.14 Baseline data for the right knee angle (black), pressure sensor set 2 (blue) and set 3 (red) are shown. Selected lock angle must occur at least $15 \mathrm{~ms}$ later than gait events and the lock can be engaged $60 \mathrm{~ms}$ after the magnetic encoder reads this angle. . . . . . . . . . . . 26

3.15 A subject in the late swing phase. . . . . . . . . . . . . . 28

3.16 Baseline right knee angle for (a) subject 6 and (b) subject 4 . Subject 4 does not flex the knee during the stance phase so the extension lock cannot be disengaged once it is engaged in the late swing phase. 31

3.17 A subject in stance phase. . . . . . . . . . . . . . . . 31

3.18 Flexion lock in the stance phase induced by SKB. Right knee angle (dashed), pressure sensor set 1 (blue) and set 4 (red) are shown. . . 32

3.19 A subject in stance phase. . . . . . . . . . . . . . . . 33

3.20 Extension lock in the stance phase induced by SKB. Right knee angle (dashed), pressure sensor set 1 (blue) and set 4 (red) are shown. . . . . . . . . . . . . . . . . 34

4.1 A four degree-of-freedom sagittal plane biped model of human standing on left leg and right leg. The model assumes that the heel of the stance leg remains in contact with the ground until the heel strike of the other leg. The hip and knee segments have distributed mass and the trunk is represented by a point mass at the hip joint.

4.2 Kinematic data (a) and kinetic data (b) for the right knee and similar data for the left knee joints (c), (d). Normal (blue) and perturbed (red) data are shown. Predicted applied torque (dashed) is obtained with the best parameters $K_{p i}$ and $K_{d i}$ of the independent joint control. . . . . . . . . . . . . . . . . . . 43

4.3 Kinematic data (a) and kinetic data (b) for the right hip joint and similar date for the left hip joint (c), (d). Normal (blue) and perturbed (red) data are shown. Predicted applied torque (dashed) is obtained with the best parameters $K_{p i}$ and $K_{d i}$ of the independent joint control. . . . . . . . . . . . . . . . . 44 
4.4 Kinetic data for the knee (a), (b) and for the hip joints (c), (d). Normal (blue) and perturbed (red) data are shown. Predicted applied torque (dashed) is obtained with the best parameters $K_{p i}$ and $K_{d i}$ of the coordinated joint control. . . . . . . . . . . . . . . . 46

4.5 Kinetic data for the knee (a), (b) and for the hip joints (c), (d). Normal (blue) and perturbed (red) data are shown. Predicted applied torque (dashed) is obtained with the best parameters $K_{p i}$ and $K_{d i}$ of the independent joint control with time shift. . . . . . . . 48

4.6 Kinetic data for the knee (a), (b) and hip joints (c), (d). Normal (blue) and perturbed (red) data are shown. Predicted applied torque (dashed) is obtained with the best parameters $K_{p i}$ and $K_{d i}$ of the coordinated joint control with time shift. . . . . . . . . . . . . . 50

4.7 Experimental setup - A subject wears SKB on the right leg while walking on a treadmill. A safety harness is used during experiments to protect subjects from falling and avoid possible injuries. . . . . . 52

4.8 Controller parameters Kp (position constant) and Kd (velocity constant) for younger and older subjects. Scatter of the parameters indicate the susceptibility of the subject to the perturbations of the gait. . . . . . . . . . . . . . . . 55

4.9 Controller parameters of three younger and three older healthy subjects involving the first stride after the disengagement of the lock. Kp, Kd and time shift values may be used to characterize subjects who are more prone to falling under gait perturbations. . . 56

5.1 Peak joint angles before and after the engagement of the flexion lock in early swing phase. Lock is engaged on one occasion. First data bar indicates the average of 10 strides before locking. Engagement of the lock reduces knee flexion and hip extension while increases hip flexion but the subjects retain their baseline within one stride after the disengagement of the lock. . . . . . . . . . . . . 58

5.2 Step length is shown for the right leg and the left leg for the average of 10 strides before the engagement of the lock and 5 strides following the disengagement of the lock. Subjects tend to take a shorter step with the right leg while they take a longer step with the left leg due to the perturbation applied to the right knee joint. . . . 59 
5.3 Normalized stride durations for the average of 10 strides before the engagement of the lock and 5 strides following the disengagement of the lock. Left swing, left double support and right double support durations decrease while right swing duration increases with the engagement of the lock. . . . . . . . . . . . . . .

5.4 Peak joint angles before and after the engagement of the flexion lock in early swing phase. Lock is engaged at every stride for five minutes. First data bar indicates the average of 10 strides before locking. Engagement of the lock reduces knee flexion and hip extension while increases hip flexion. . . . . . . . . . . . . 61

5.5 Peak joint angles before and after the disengagement of the flexion lock in early swing phase. First data bar indicates the average of 10 strides before the disengagement of the lock. Second data bar shows the baseline peak joint angles for pre-locking condition. Hip flexion increases significantly following the release of the lock. . . . . . . 62

5.6 Step length is shown for the right leg and the left leg for the average of 10 strides before the engagement of the lock and the first 5 strides of continuous locking. Subjects tend to take a shorter step with the right leg while they take a longer step with the left leg due to the perturbation applied to the right knee joint. . . . . . . . . . .

5.7 Step length before and after the disengagement of the flexion lock in early swing phase. First data bar indicates the average of 10 strides before the disengagement of the lock. Second data bar shows the baseline step length for pre-locking condition. An overshoot in both right and left step lengths is observed with the disengagement of lock at the end of five minutes. . . . . . . . . . . . . . . . . . 64

5.8 Normalized stride durations for the average of 10 strides before the engagement of the lock and the first 5 strides of continuous locking. Double support durations decrease while swing durations increase with the engagement of the lock. . . . . . . . . . . . 65 
5.9 Normalized stride durations before and after the disengagement of the flexion lock in early swing phase. First data bar indicates the average of 10 strides before the disengagement of the lock. Second data bar shows the baseline duration for pre-locking condition. Subjects adapt a longer right swing duration while left swing, left double support and right double support durations decrease at the end of five minutes. . . . . . . . . . . . . . . . . . . . 66

6.1 Lock mechanism with separate extension and flexion gear plates. . . 68 


\section{LIST OF TABLES}

4.1 EXPERIMENTAL PROTOCOL ............... . . 53 


\begin{abstract}
Smart Knee Brace (SKB) is designed to provide controlled perturbations to the human knee during walking. A motion capture system records the gait movement before and after these perturbations to assess the human response to the perturbations. A dynamic model of human walking is then used to evaluate the human applied joint torques to hypothesize how the human neuro-muscular system modulates the joint torques as a response to the perturbations caused on the gait.

Subjects respond differently to perturbations but all healthy subjects restore their nominal gait in a few subsequent cycles. With a single perturbation to the gait, the subjects restore their nominal gait immediately while they tend to show aftereffects under sustained perturbations. The results show that the neuro-muscular response to perturbations can be reasonably well characterized by including the following features in the model: (i) nominal gait in the absence of perturbation, (ii) corrective torque at a joint in response to the error at that joint and other joints, (iii) a characteristic time shift in the response. There is intuitive justification for each of the components included in the model. Detailed parameters that characterize these components, obtained by data fitting from the response data, are specific to an individual's neuro-muscular response to perturbations in the gait. These parameters may be used to characterize subjects who are more prone to falling under gait perturbations.
\end{abstract}




\section{Chapter 1}

\section{INTRODUCTION}

The Smart Knee Brace (SKB) allows physical therapists the ability to research, develop and implement effective training practices for gait rehabilitation through motion learning and feedback to the human neuromuscular system. In this study, the SKB is used to develop a new method to categorize subjects that are prone to falls and to induce righting reactions and longer term changes in kinematic patterns that might be useful in preventing falls.

\subsection{The Effects of Fall}

Today, the number of people over the age of 60 is growing rapidly and is estimated to reach 2 billion worldwide by 2050 [6]. One third of older adults living in the community falls at least once a year [4, 22]. The consequences of falls include reduced quality of life and independence [4], physical and psychological trauma [5], functional deterioration [17], substantial health care costs [29, 25, 17], increased morbidity and mortality [24]. Therefore, it is timely to focus on methods and interventions that can quantify and prevent individuals from falling. Human body is an inherently unstable system with its distribution of mass due to neurological deterioration with age, falls become even more important issue for the elderly [31].

Studies have shown that falls are neither accidental nor random events but are predictable occurrences [5]. A number of studies have demonstrated fall predictors including asymmetry in kinetics of the limbs [27], reduced lower limb strength [23], weakened handgrip strength [28], impairments in postural stability, reaction time 
and visual functions [4]. Falls can be reduced or prevented by reducing the risk factors and by proper training [7]. Studies suggest that correcting kinematic errors in gait has an important role in motor learning [32], so it is plausible that rehabilitation programs that perturb joint kinematics during walking can be used to investigate the neuromuscular control of human locomotion [19].

\subsection{Current Training Methods}

Studies suggest that proper training can contribute to fall prevention $[7,33$, 21]. Among the different training methods, perturbation paradigms are useful in studying human control of locomotion [19]. Several studies have focused on effects of phase specific perturbations applied via moving force plates [8,30,34], platforms

$[15,18]$, compliant surfaces [16], and unexpected obstacles [26, 23, 20]. Due to the nature of these paradigms, often subjects are able to quickly figure out when and where to expect these perturbations and prepare themselves for a forthcoming event. In some studies, electrical stimulation was used to perturb the human gait [35]; however, it is debatable if activations of cutaneous or proprioceptive afferents occur in realistic perturbations in daily life [26]. In some studies, the timing of the perturbation becomes hard to precisely control [16] and obstacles become visible to the subjects [9]. Today, very little is known about how humans will respond to perturbations in the human gait when their knees are locked at predetermined angles.

\subsection{Smart Knee Brace Concept}

Humans have an unusual acumen to respond to unpredicted perturbations. Unpredictability should be an important requirement in the design of experiments while studying gait recovery from perturbations since the central nervous system (CNS) can quickly learn and adapt to these external stimuli [21]. Adapting to the environment and motor learning is a crucial function for the CNS, hence, locomotor 
adaptations of the CNS to changes in the walking condition have been investigated in numerous studies $[10,11,12,13,14]$.

Smart Knee Brace (SKB) is an in-house developed power brace which can apply controlled perturbations to the human knee during the gait cycle such that subjects do not know when to expect a perturbation. The computer interface of SKB allows locking of the knee joint during swing or stance at a desired angle. The knee joint can be prevented from flexing or extending while a subject is walking.

\subsection{Summary and Thesis Outline}

This thesis presents the description of an in-house designed brace labeled smart knee brace SKB, to investigate neuro-muscular responses to perturbations in gait that induce errors in lower-limb kinematics. Joint kinematics are then utilized by a dynamic model of the human leg to investigate neuro-muscular response to these perturbations through various error correcting models and least square data fitting.

Chapter 2 details the features of the SKB. Functionally, it can lock and unlock the knee joint at an arbitrary point during the gait cycle.

Chapter 3 discusses the improvements on the hardware and software parts of the device. The brace hardware is modified to increase the subject pool and the software is improved in order to properly regulate and condition the sensors for locking mechanism on the brace.

In Chapter 4, a dynamic model of human walking is outlined. This model is used to evaluate the human applied joint torques to hypothesize how the human neuro-muscular system modulates the joint torques as a response to the perturbations caused on the gait.

Chapter 5 analyzes the kinematic responses of young and older healthy subjects to perturbations made via the SKB.

Chapter 6 presents a summary of the work and discusses future work. 


\section{Chapter 2}

\section{SMART KNEE BRACE}

SKB is a knee ankle foot orthosis (KAFO), and can be used to lock and unlock the knee while a subject is walking [1]. The shank segment of the brace can be adjusted so that the brace can be used on patients of varying heights. The SKB knee joint is uniaxial and can flex $110^{\circ}$ and extend to $25^{\circ}$ hyperextension. The ankle joint can move freely. The knee joint of the brace is fitted with a specially designed gear and a lock mechanism, as shown in Figure 2.1. This lock is powered by a linear solenoid which engages and disengages a lock pawl into the gear plate. The flexion lock operates within the range $-55^{\circ}$ and $20^{\circ}$, where $0^{\circ}$ represents a neutral knee ( $180^{\circ}$ between the thigh and shank segments), negative angle represents flexion and positive extension beyond a neutral knee position. The extension lock operates over the range $-30^{\circ}$ and $20^{\circ}$.

In order to achieve locking the knee joint at an arbitrary point during the gait cycle, SKB uses pressure sensors and a magnetic encoder. Twelve pressure sensors are placed on the sole of the shoe along its length, divided into four sets, which effectively introduce four pressure zones to be used in the control algorithm. Each set has three sensors, placed laterally, centrally, and medially to achieve full coverage of the foot.

The angular position of the knee joint is determined by a miniature absolute magnetic shaft encoder. The shaft of the encoder is fixed on the brace frame while its body is fixed on the gear plate. Thus, the axis of rotation of the encoder is aligned with the axis of rotation of the knee joint. 


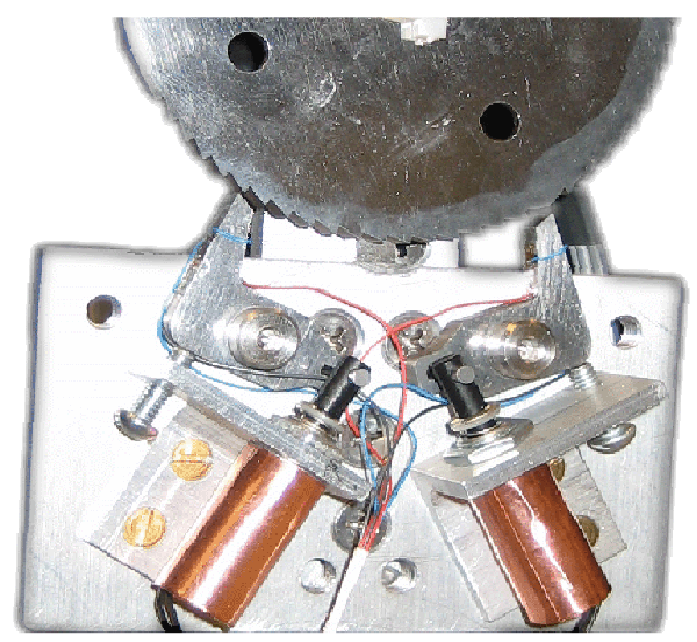

Figure 2.1: The locking mechanism. Each lock is powered by a solenoid that engages and disengages a lock pawl into a gear plate. The locks function independent of each other.

The computer integration of SKB is realized via a host and a target computer. The therapist controls the host computer, which has Matlab and XPC Target Toolbox installed on it. The target computer is crucial for real-time control of the brace and it is controlled by the host computer. Customized user-friendly software, with a graphical user interface is developed in order to facilitate data collection throughout experiment sessions. The circuit box depicted in Figure 2.2 supplies power and amplifies sensor signals so that they could be picked via data acquisition card. Pressure sensor and knee angle data picked by the brace are received by the target computer, which processes and transmits lock information to the brace in real-time. Lock information includes the details about the locking strategy and will be discussed in further detail in the next chapter. 


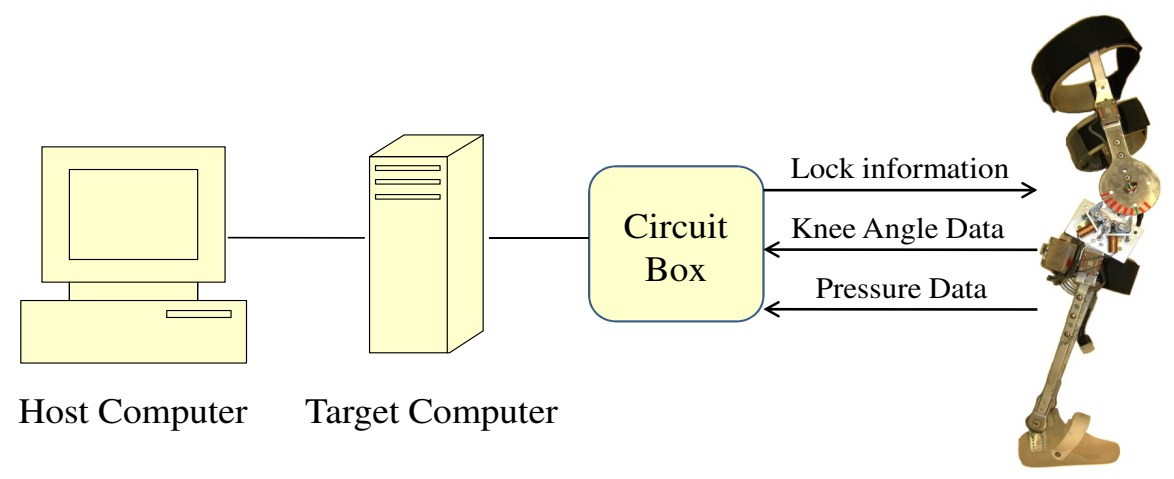

SKB

Figure 2.2: The components and computer interface of SKB. Right knee joint angle data and pressure from four sets of sensors underneath the foot are sent by SKB. The brace receives the details of the locking condition such as lock angle, lock duration and number of engagements. 


\section{Chapter 3}

\section{HARDWARE ADAPTATIONS AND THE CONTROL ALGORITHM}

The effectiveness of the SKB in evaluating training techniques and the ability to lock the knee joint at specific angles and phases in the gait cycle are highly dependent upon the accuracy of the sensors on the brace. Previous work has been performed to validate the sensors used in the data collection on the brace [1]. However, experiments indicated a considerable amount of difference between the lock angle set through the software and the actual knee angle at which the locks got engaged. This inconsistency led to three different angle definitions: (i) lock angle, (ii) brace angle, and (iii) actual joint angle. Lock angle is the desired angle to engage the brace lock and it is set through the software. Brace angle is the current angular position of the brace knee joint and detected by the magnetic encoder. Actual joint angles are provided by the motion capture system. Reflective markers are placed on the subjects in order to record joint kinematics via an 8-camera Vicon motion analysis system at a sampling rate of $100 \mathrm{~Hz}$. Actual joint angle is taken as a basis while diagnosing hardware and software problems of the brace. As far as the previous experimental data are concerned, these three angles are observed to be different from each other in each trial. Furthermore, a consistent correlation between these angles could not be observed. For the brace to function effectively during experiments, the setbacks of the previous design are resolved by means of improvements on the brace hardware and software. A new control algorithm, which 
takes the limitations of the device into account is developed. This will be described in detail in this chapter.

\subsection{The Hardware}

The hardware of the SKB is improved in order to (i) make subjects more comfortable with the brace, (ii) prevent subject's leg from moving inside the device, (iii) increase the accuracy of sensor readings, and (iv) create a consistent correlation between the angles discussed above. The reason for the difference between the lock angle and the brace angle is principally attributed to the electronics and software parts of the system and investigated in detail in Section 3.2 but it is also deteriorated by the rigid frame of the brace. The SKB is a custom-fit device designed for a specific subject. The brace cannot fit each subject perfectly given that anthropometric measures vary from one subject to another. To minimize this effect, subjects are selected with appropriate sizes that can fit in the brace.

Even though the shank segment of the brace can be adjusted for different lengths, individuals whose shank segments are shorter than 15.7 inches cannot fit in the brace. In addition to segment length, segment width is also a crucial criterion for subject selection. If the width of the shank or thigh segments of a subject is larger than the maximum allowable width of the corresponding segments on the device, then misalignment becomes an issue. Once this is the case, there is an offset in the knee angle reading of the magnetic encoder. If tight fit is provided and the leg does not move inside the brace, offset in the angle reading maintains a constant value so that experiments could be run by taking this offset into account while engaging knee locks. On the other hand, padding must be used if the segment width of the subject is smaller than the corresponding width on the brace. The gap between the brace and subject's leg is filled with padding in order to prevent the leg from moving inside the brace and to improve the fit. 
In addition to padding, replacing the ankle segment with an adjustable part curtails the movement of the leg inside the device significantly. Previous ankle segment was a rigid aluminum part and failed to grab the foot. Furthermore, joint position was fixed on the brace due to the custom design. This condition further worsened the misalignment problem because it was difficult to align the human ankle joint with the ankle joint on the brace. On the contrary, new ankle segment is a plastic shoe insert, which can grab the foot tightly. Also, with the adjustable joint position, the new segment enables the therapist to align the ankle joint on the brace with the ankle joint of the subject. Once the ankle joints are aligned, knee joints can also be aligned properly by adjusting the height of the shank segment on the brace. As a result, the movement of the leg inside the brace is minimum as much as possible, which ensures consistency of experimental results across different subjects. Therefore, the therapist can always be aware of the actual knee joint angle as well as the brace angle, while setting the lock angles in the software.

Figure 3.1 illustrates the improvement of brace fit. In the figure, solid blue line (brace angle) shows the reading of the magnetic encoder and solid red line (actual joint angle) shows the reading of the motion capture system. Because it is impossible for any orthotic device to reproduce the relative movements between the bones in the thigh and shank segments perfectly, an offset exists between the knee angle data obtained from the brace and the same data computed via the motion capture system. Once the offset is taken into account, the adjusted brace angle deviates from the human knee joint angle characterized by the motion capture system by no more than $0.5^{\circ}$.

Figure 3.2 shows peak hip and knee joint angles for the right leg as well as the left leg. As it is possible to observe in Figure 3.2 (a) and (b), peak joint angles for the right and the left leg are not identical before the improvements on the hardware owing to joint misalignment and discomfort of the brace. On the other hand, peak 


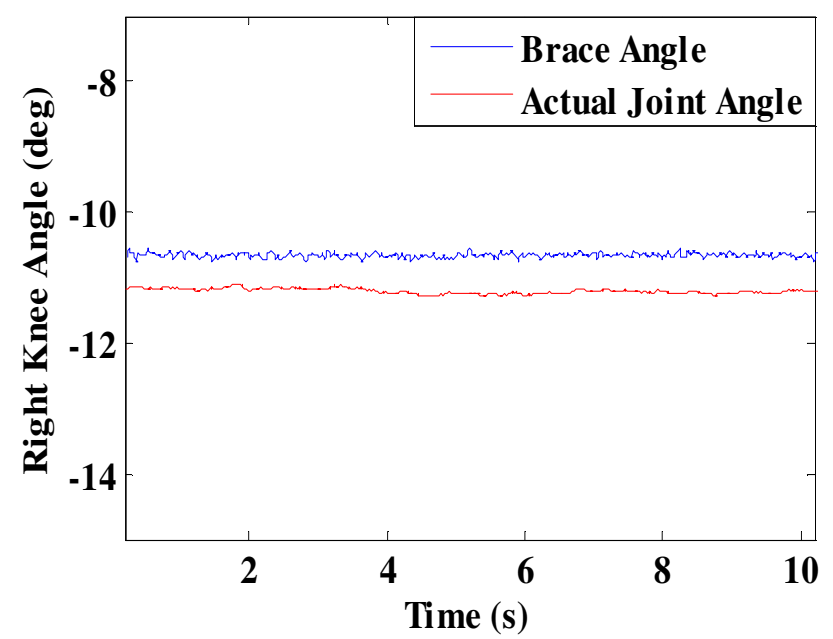

Figure 3.1: Right knee joint angle data is collected via the brace (blue) and motion capture system (red) while the subject is standing still on the treadmill. The difference between two lines is no more than $0.5^{\circ}$ and consistent throughout the experiment.

joint angles converge after the improvements on the brace hardware, as shown in Figure 3.2 (c) and (d).

Pressure sensor readings are improved with the parallel connection of a third sensor to each of the pairs. As a result, twelve pressure sensors are placed underneath the foot along the length, divided into four sets. Each set has three sensors connected in parallel and they are placed laterally, centrally, and medially to achieve full coverage of the foot.

\subsection{The Software}

The ability to lock the knee joint at specific angles and phases in the gait cycle depends upon proper data acquisation and processing. Investigation of data collected through experiments indicated that the limitations of the brace must be taken into consideration in order to have a reliable lock trial. Diagnosis of the 
limitations of the device and the development of the new control algoritm under these limitations are addressed in further detail in this section. 


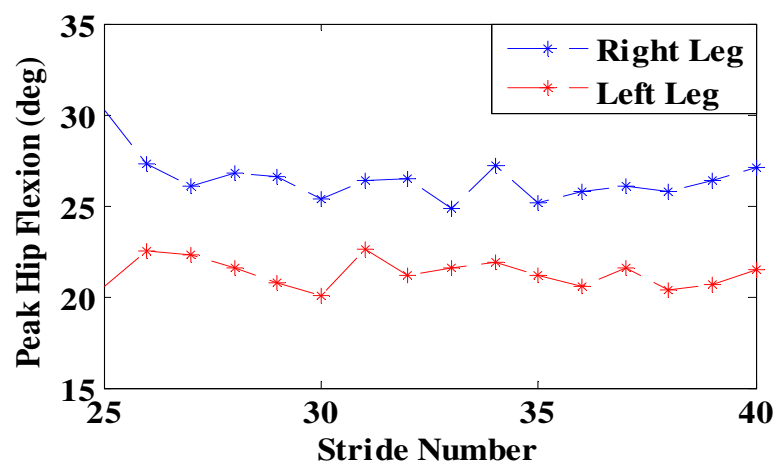

(a)

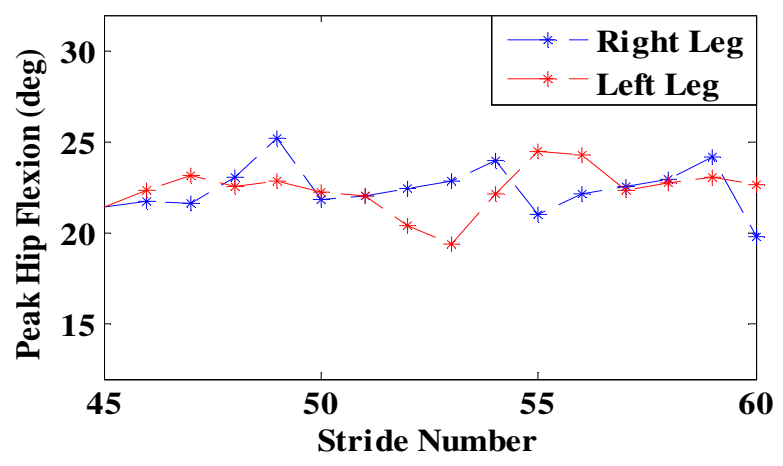

(c)

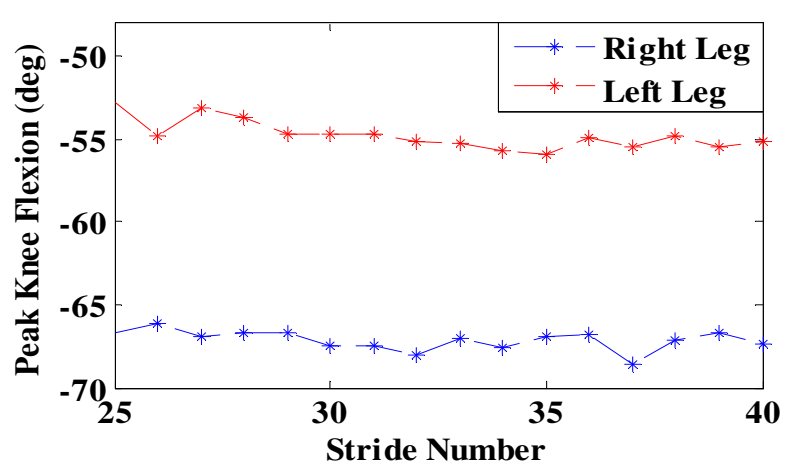

(b)

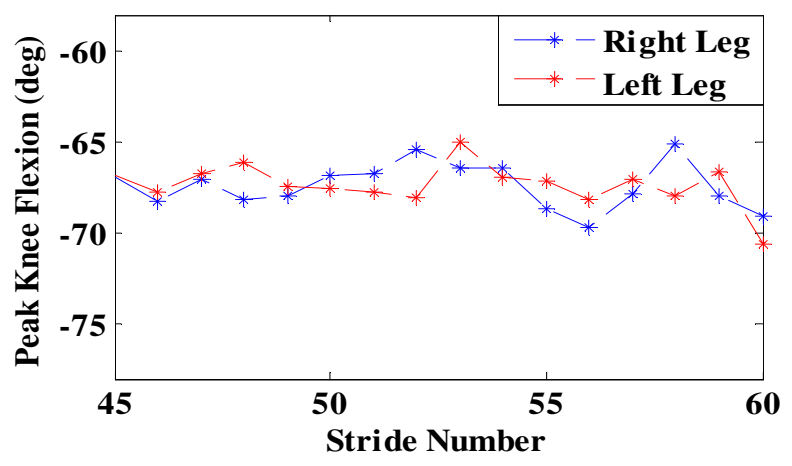

(d)

Figure 3.2: Peak joint angles for the right (blue) and left (red) leg are shown. Before hardware improvements, (a) hip angles and (b) knee angles differ from each other approximately by $10^{\circ}$. Hardware improvements have a significant influence on (c) hip and (d) knee data, which also indicates subject's comfort with the brace. 


\subsubsection{Built-in Time Delay within the System}

Data collection sessions showed that the predetermined lock angle was never identical to the angle at which the locks got activated. Further investigation proved that there is a built-in time delay, which is due to both hardware and software parts of the system. Basically, the time delay is caused by the processing time of the software as well as the activation time of the solenoid to engage the lock. In the following sections, time delay is analyzed in further detail. We found out that it does not take the same amount of time for the solenoid to get activated in response to the readings of the magnetic encoder and pressure sensors.

\subsubsection{Time Delay in Response to Pressure Sensor Data}

A small switching circuitry is built on the gear mechanism to be able to measure the exact duration between the time at which the signal is sent to engage the lock, and the time at which the lock gets activated. As soon as the lock gets engaged, the circuit is closed and an input voltage of $2 \mathrm{~V}$ is supplied to the analog input port of National Instruments Data Acquisition card. In the previous studies, this time delay was found to be $10.3 \mathrm{~ms}$; however, experimental results indicated a higher value than 10.3 ms. Further inspection proved that time delay is closely related to the sampling rate of data collection with the Target Computer. For this reason, several data were collected with various sampling rates so as to find out the time delay accurately. Figure 3.3 shows the signal that is sent to engage the lock, and the response of the solenoid to this signal when the sampling rate of the Target Computer is $50 \mathrm{~Hz}$.

The time delay within the system seems to be $40 \mathrm{~ms}$ for a sampling rate of 50 Hz. In the figure, solid blue line shows the reading of the heel pressure sensor. As soon as the heel pressure sensor reading reaches a threshold value, which is set as 25

lbs in this specific example, the signal is sent to activate the lock. Upon receiving the signal to activate the lock, the flexion lock gets engaged. When the flexion lock 


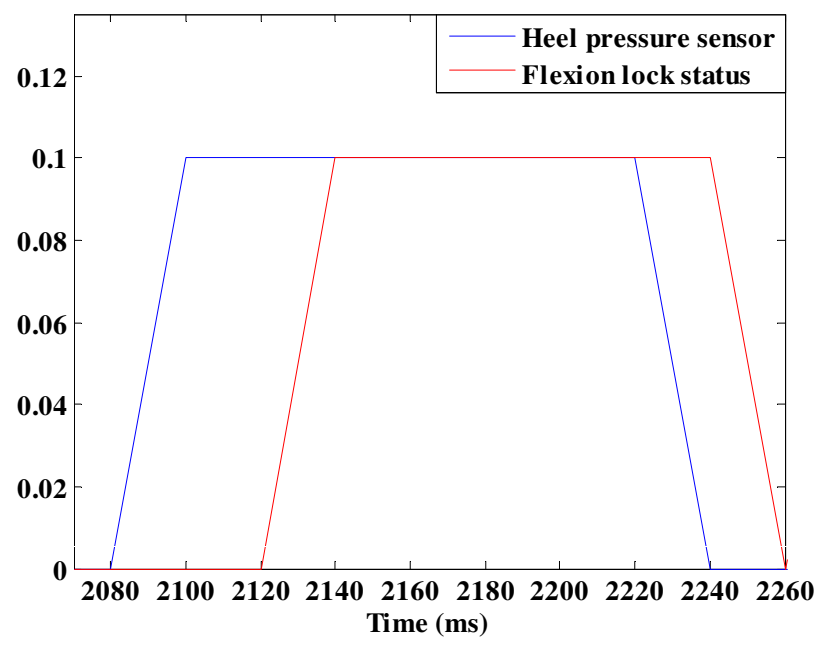

Figure 3.3: Heel pressure sensor data (blue) and flexion lock status (red) are shown. Threshold value of the heel pressure sensor and the status of the flexion lock are scaled to the same constant value. The time delay within the system seems to be $40 \mathrm{~ms}$ when the sampling rate of the Target Computer is $50 \mathrm{~Hz}$.

gets engaged, the circuit on the gear mechanism is closed and an input voltage of $2 \mathrm{~V}$ is supplied to the analog input port. Therefore, the status of the lock can be detected upon receiving the input voltage as depicted by the solid red line in Figure 3.3. For convenience, the threshold value of the heel pressure sensor and the status of the flexion lock are scaled to the same constant value in the figure. Ideally, as soon as the heel pressure sensor reading reaches the threshold value, the lock must get engaged at the very same moment. However, Figure 3.3 illustrates that signals do not reach their peak values at the same instant. Therefore, one can conclude that it takes $40 \mathrm{~ms}$ for the lock to get engaged after receiving the signal for a sampling rate of $50 \mathrm{~Hz}$. In order to demonstrate the dependency of the time delay on the sampling rate of data collection, data are collected with several sampling rates, and Figure 3.4 depicts the response of the lock with a sampling rate of $100 \mathrm{~Hz}$.

When the sampling rate is set to $100 \mathrm{~Hz}$, the time delay is observed to be 30 


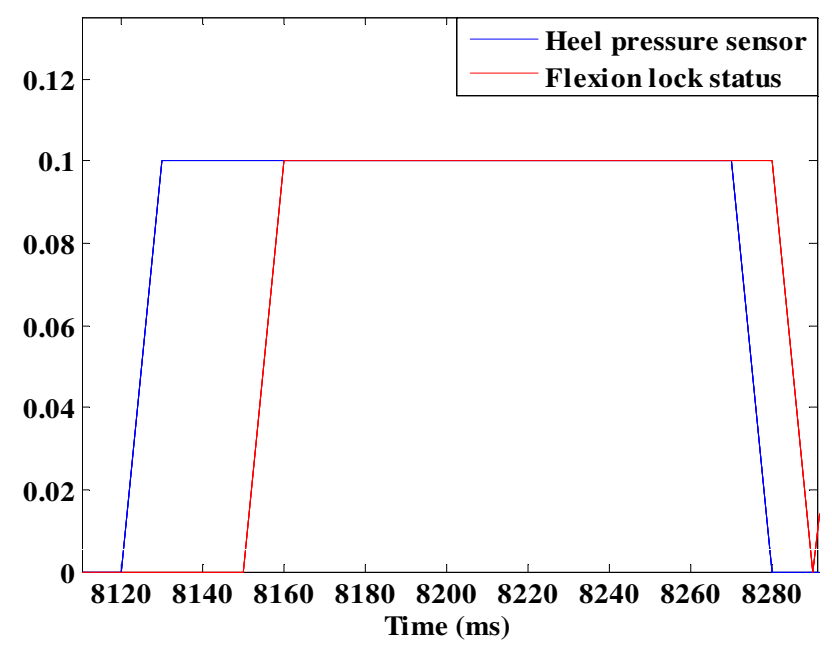

Figure 3.4: Heel pressure sensor data (blue) and flexion lock status (red) are shown. The time delay within the system seems to be $30 \mathrm{~ms}$ when the sampling rate of the Target Computer is $100 \mathrm{~Hz}$.

ms. Figure 3.5 illustrates the response of the lock with a sampling rate of $1200 \mathrm{~Hz}$.

The time delay is observed to be $15 \mathrm{~ms}$ for a sampling rate of $1200 \mathrm{~Hz}$. As it is possible to observe from figures above, built-in time delay within the system is strictly dependent on the sampling rate used for data collection. The experiment is repeated for a range of sampling rates from $50 \mathrm{~Hz}$ to $1200 \mathrm{~Hz}$ and time delay value converges to $15 \mathrm{~ms}$ as the sampling rate increases. For sampling rates with low frequencies such as $50 \mathrm{~Hz}$, the time delay cannot be measured accurately because the time delay itself is smaller than the sampling time. When the sampling rate is set to $50 \mathrm{~Hz}$, data is sampled at each $20 \mathrm{~ms}$. However, the actual time delay is observed to be $15 \mathrm{~ms}$. On the other hand, when the sampling rate is set to 1000 $\mathrm{Hz}$, data is sampled at each $1 \mathrm{~ms}$. To be able to measure the time delay accurately, time delay itself must be smaller than the time duration between two consecutive sampling points. For this reason, during experiment sessions, a sampling rate of 600 $\mathrm{Hz}$ is used. Therefore, one has to bear in mind that the knee lock gets engaged 15 


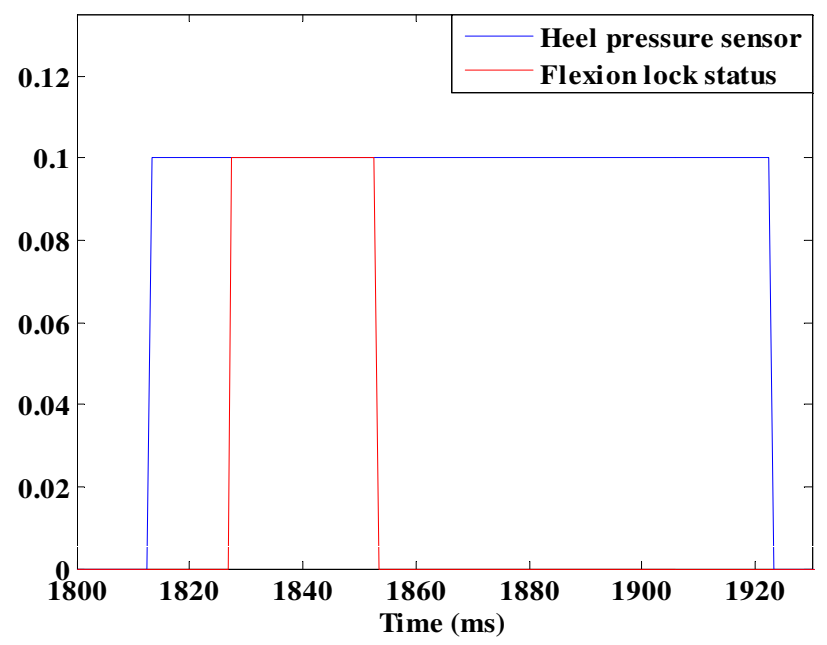

Figure 3.5: Heel pressure sensor data (blue) and flexion lock status (red) are shown. The time delay within the system seems to be $15 \mathrm{~ms}$ when the sampling rate of the Target Computer is $1200 \mathrm{~Hz}$.

ms after the pressure sensor reading reaches its threshold value.

\subsubsection{Time Delay in Response to Magnetic Encoder Data}

The latest control algorithm takes advantage of the magnetic encoder as well as pressure sensor data in order to engage knee locks. In Section 3.2.1.1, time delay in response to pressure sensor is analyzed in detail and it is concluded that if the sampling rate is $600 \mathrm{~Hz}$, then the time delay must be $15 \mathrm{~ms}$. However, further investigation of experimental data revealed that the engagement of knee locks takes more than $15 \mathrm{~ms}$ even though the sampling rate is set to $600 \mathrm{~Hz}$. The extra time delay within the system can only be explained by a time delay in response to the magnetic encoder data.

Figure 3.6 illustrates a trial in which the flexion lock is engaged during earlyswing phase. The lock is set to engage when knee flexion angle reaches $25^{\circ}$; however, as can be seen in the figure, the lock is engaged at $37^{\circ}$ of flexion. When the time difference between these two points is taken into consideration, one can observe that 
it takes $60 \mathrm{~ms}$ for the flexion lock to get engaged after the magnetic encoder reading reaches the prescribed angle value.

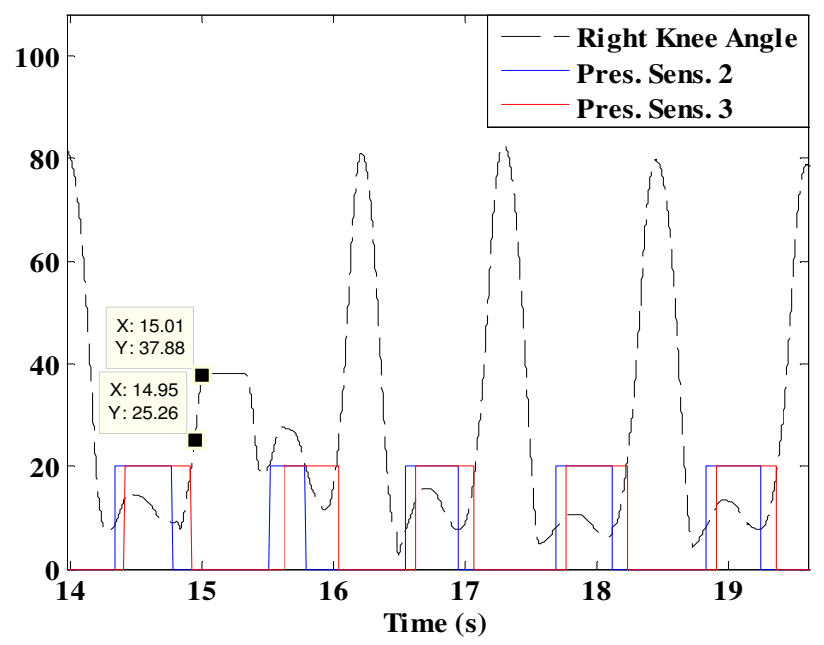

Figure 3.6: Right knee angle (dashed), pressure sensor set 2 (blue) and pressure sensor set 3 (red) are shown. It takes $60 \mathrm{~ms}$ for the flexion lock to get engaged after the magnetic encoder reading reaches the preset angle value.

Figure 3.7 illustrates another trial in which the extension lock is engaged in the late swing phase. The lock is set to engage when knee extension angle reaches $40^{\circ}$ but it is engaged at $18^{\circ}$ of extension. Time difference between these two data points is measured as $60 \mathrm{~ms}$. As a result, repeating the experiment across different lock conditions indicated the same amount of delay, which is attributed to the activation of the solenoid in response to the magnetic encoder data.

\subsubsection{Control Algorithm Based On Pressure Sensor Data}

As far as the preliminary control algorithm is concerned, data collected through experiment sessions were not satisfactory due to the sporadic manner of lock engagements. Processing the data revealed that activating knee locks at a desired angle does not work for every subject. Each individual has a unique walking 


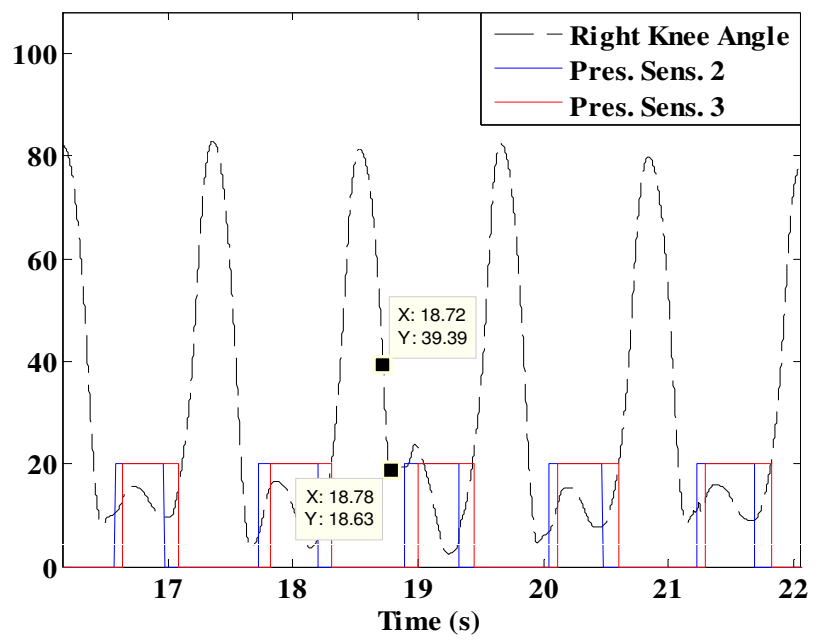

Figure 3.7: Right knee angle (dashed), pressure sensor set 2 (blue) and set 3 (red) are shown. It takes $60 \mathrm{~ms}$ for the extension lock to get engaged after the magnetic encoder reading reaches the preset angle value.

pattern and this fact has to be taken into consideration before trying to engage the knee locks. For instance, the flexion lock can be engaged at $30^{\circ}$ for a certain subject; however, this might not be feasible for another subject with the previous algorithm. As a result, a new concept, activating knee locks a certain amount of time after gait events, is introduced in this section.

First of all, the subject is allowed to walk on the treadmill with the brace for 5 minutes at a certain treadmill speed. This period is called the acclimation period, which helps the subject get used to walking with the brace. At the end of the acclimation period, baseline gait pattern of the subject is collected via the brace. As shown in Figure 3.8, baseline pattern includes angle data from the magnetic encoder and all four sets of pressure sensors. Baseline walking pattern of the subject is analyzed to determine the convenient points in the gait cycle at which knee locks could be engaged.

In the figures, pressure sensor signals are scaled to 20 if the signal is above the 


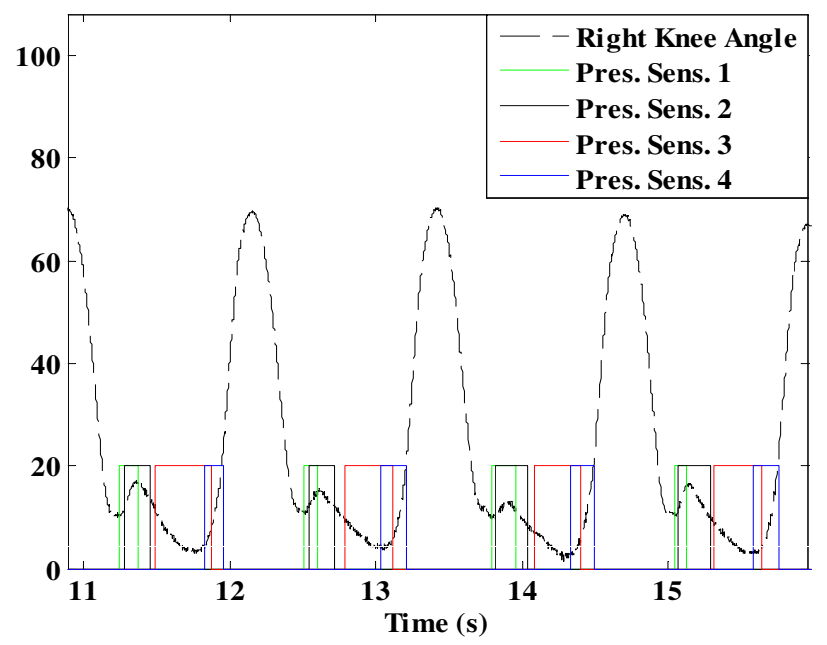

Figure 3.8: Right knee angle (dashed), pressure sensor set 1 (green), set 2 (black), set 3 (red) and set 4 (blue) are shown on the baseline gait pattern of a subject. The baseline pattern is analyzed to determine the convenient points in the gait cycle at which knee locks could be activated.

threshold and to 0 if it is below the threshold. First, heel-strike and toe-off events are detected on the baseline. Heel-strike event occurs as soon as Pressure Sensor Set 1 reading exceeds the threshold value. Toe-off event occurs when Pressure Sensor Set 4 reading falls below the threshold value. Second, the average time duration from heel-strike and toe-off to the desired lock angle is measured. To this purpose, an arbitrary knee flexion angle is selected, $35^{\circ}$ in this specific trial. Next, duration from heel-strike and toe-off to the predetermined angle is measured for every single stride in the baseline. Then, these numbers are averaged by stride number, and thus an average time from heel-strike to the prescribed lock angle and an average time from toe-off to the same angle are calculated. In this trial, it takes on average 747 $\mathrm{ms}$ for the subject to get to $35^{\circ}$ of flexion from heel-strike and $25 \mathrm{~ms}$ from toe-off to the same angle. As discussed in Section 3.2.1.1 in detail, knee locks have a time delay of $15 \mathrm{~ms}$ in response to pressure sensor data. Therefore, in order to engage the flexion lock at $35^{\circ}$, the lock must be activated $732 \mathrm{~ms}$ after the heel-strike or 
$10 \mathrm{~ms}$ after the toe-off. Figure 3.9, shows the engagement of the flexion lock $10 \mathrm{~ms}$ after the toe-off event.

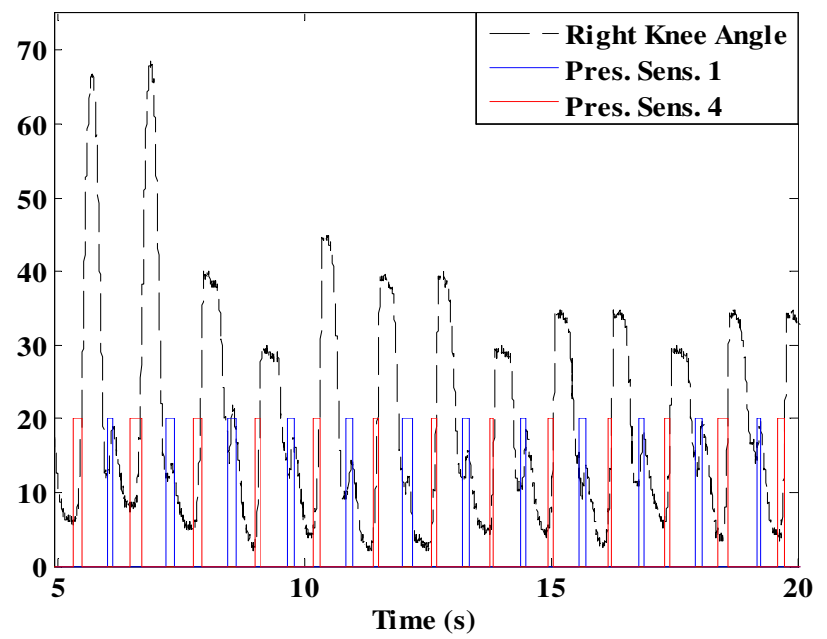

Figure 3.9: Engagement of flexion lock $10 \mathrm{~ms}$ after toe-off. Pressure Sensor Set 1 (blue) data is used for heel-strike detection while Pressure Sensor Set 4 (red) is used for toe-off detection. Magnetic encoder data (dashed) shows right knee angle.

As can be seen in Figure 3.9, after $\mathrm{t}=8$ second, the lock gets engaged in every single stride as desired. As opposed to the previous control algorithm, this strategy ensures that the lock gets engaged for each individual subject. On the other hand, one has to keep in mind that the lock is activated $10 \mathrm{~ms}$ after the toe-off event and this is an average value calculated across a number of strides. There may be a slight variation in time from the toe-off to the prescribed angle in each stride. This variation in time also manifests itself as a variation in the angle at which the lock gets engaged. Even though the lock is aimed to get engaged at $35^{\circ}$ of flexion, there is a variation in lock angle from $25^{\circ}$ to $40^{\circ}$ across different strides. So as to realize the variation in time across different strides, knee lock is activated $0 \mathrm{~ms}$ and $15 \mathrm{~ms}$ after the toe-off event. Figure 3.10 and Figure 3.11 illustrate the results for these conditions. 


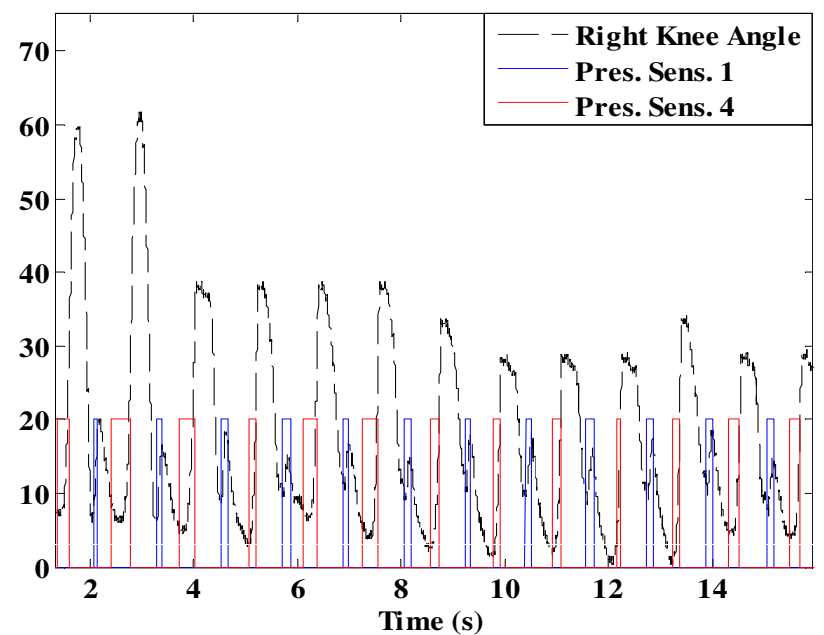

Figure 3.10: Engagement of flexion lock $0 \mathrm{~ms}$ after toe-off. Pressure Sensor Set 1 (blue) data is used for heel-strike detection while Pressure Sensor Set 4 (red) is used for toe-off detection. Magnetic encoder data (dashed) shows right knee angle.

As can be seen in figures above, in each of the trials the lock can be engaged at $35^{\circ}$ of flexion. In other words, it may take $0 \mathrm{~ms}$ for the subject to get to the predetermined flexion angle from the toe-off event in one stride while it may take $15 \mathrm{~ms}$ in another stride. As can be observed in Figure 3.11, the flexion lock did not get engaged at time $t=13$. A closer look at the figure reveals that pressure sensor set 4 reading is missing in that specific stride. For this reason, the algorithm was not able to detect the toe-off event and hence the lock did not get engaged. In this strategy, only pressure sensor information is taken into account in order to activate the knee locks. For this reason, one has to make sure sensor readings are strong enough before each experiment. Once pressure sensors start losing their sensitivity, they have to be replaced by new sensors in order to maintain the reliability of the algorithm.

The main assumption in this strategy was the duration from heel-strike or toe-off event to the predetermined knee angle would not show drastic deviations 


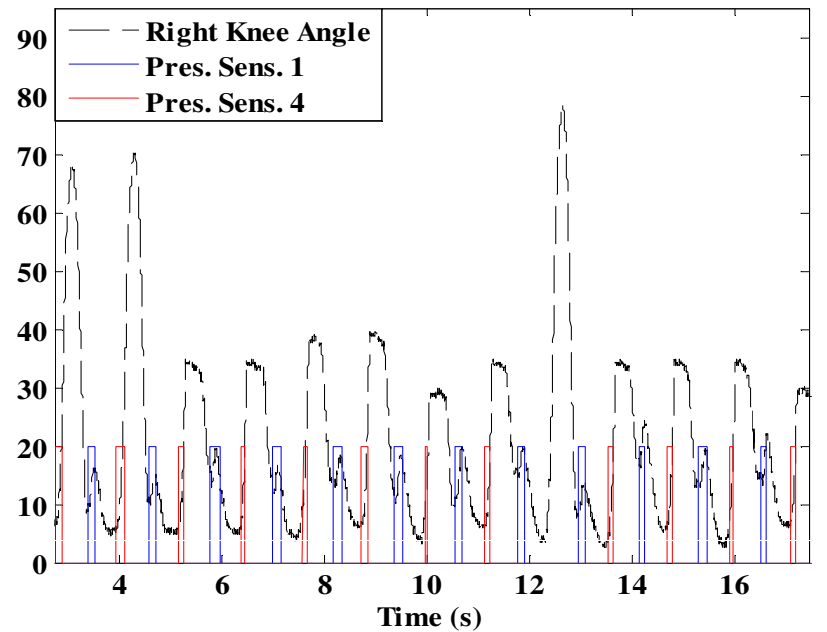

Figure 3.11: Engagement of flexion lock $15 \mathrm{~ms}$ after toe-off. Pressure Sensor Set 1 (blue) data is used for heel-strike detection while Pressure Sensor Set 4 (red) is used for toe-off detection. Magnetic encoder data (dashed) shows right knee angle.

from an average value, which can be derived from the baseline. Experimental data indicated that perturbations due to the engagement of locks change the baseline of subjects. Therefore, the knee is locked at different angles in each trial due to these perturbations. The main disadvantage of the algorithm is the inconsistency in the angle value at which the lock gets engaged. When the heel-strike event is used as the reference to activate the locks during the swing phase, more variations are observed in terms of lock angles. For instance, it takes on average $747 \mathrm{~ms}$ for this subject to get to the preset angle value from heel-strike while it takes just $25 \mathrm{~ms}$ to reach the same angle from toe-off. As the duration from the event to the predetermined angle increases, more variations in time as well as lock angle across different strides are observed. For this reason, heel-strike event is preferred in order to engage knee locks during the stance phase while toe-off event is preferred to engage locks during the swing phase. On the other hand, the algorithm has a number of advantages when compared to the previous one. First, it enables the 
therapist to take into account the built-in time delay in response to pressure sensor data. Once the average duration from a gait event to the predetermined knee angle is calculated, $15 \mathrm{~ms}$ of time delay can be deduced from this average value so that the lock could be engaged at the desired angle. Second, it works for different treadmill speeds. Experiments were run with varying speeds from $1.8 \mathrm{mph}$ to $2.4 \mathrm{mph}$ and in each trial results were satisfactory. Finally, it ensures that the locks can be engaged for every single subject due to the fact that durations from gait events are calculated for each subject individually and hence unique gait pattern of each subject is taken into consideration. If the results of the experiments are desired for perturbation in gait studies regardless of the lock angle, this algorithm is highly useful. However, if the knee is desired to be locked at a certain flexion or extension angle at an arbitrary point in the gait cycle, another algorithm needs to be developed, which is discussed in detail in the next section.

\subsubsection{Control Algorithm Based On Pressure Sensor and Magnetic En- coder Data}

This strategy enables the therapist to engage the knee locks at an arbitrary point in the gait cycle, as shown in Figure 3.12. Essentially, the knee can be prevented from flexing during loading response (LRFL), locked so the knee cannot

extend in Midstance (MSEL), prevented from flexing in early swing (ESFL) or prevented from extending in late swing (LSEL).

These locking strategies can each be used separately or in combination and can be engaged for one stride of walking or continuously during each stride of walking. The points in the gait cycle along with lock activations are described in detail in the sections that follow. 


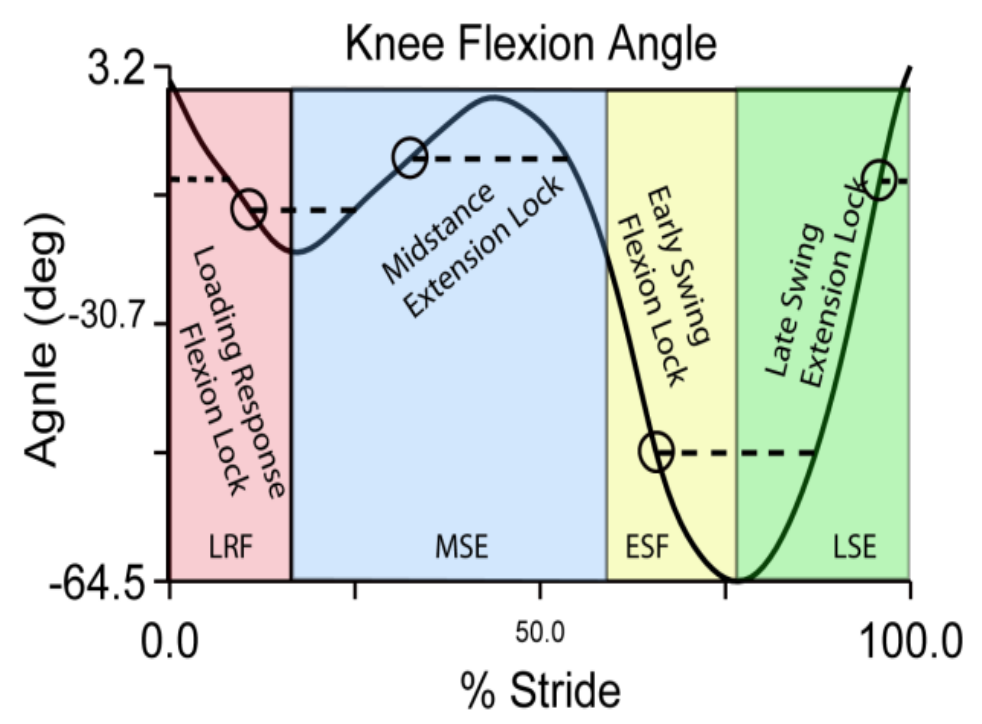

Figure 3.12: Locking strategies during one stride (heel-strike to the next consecutive heel-strike). Flexion locks can be engaged during Loading Response and Early Swing Phase, Extension Locks can engage during Midstance and Late Swing Phase at prescribed angles.

\subsubsection{Flexion Lock in Early Swing Phase}

In this strategy, the knee lock is activated when the subject flexes the knee during the early-swing phase. As seen in Figure 3.13, subject's left leg is on the ground and the right leg swings backwards during the early-swing phase. The lock gets engaged following the toe-off of the right leg and it is kept locked until the subject starts extending the knee. With the engagement of the lock, the subject experiences a major perturbation to the gait.

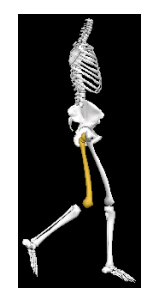

Figure 3.13: A subject in the early swing phase. 
As discussed in Section 3.2.2, experiments begin with the acclimation period in order for the subjects to get used to walking with the brace. Next, baseline gait pattern is collected so as to take the unique walking pattern of each individual subject into consideration. In this strategy, magnetic encoder data is evaluated in addition to pressure sensor data to be able to activate the knee locks at a desired angle. First of all, heel-strike and toe-off events are determined on the baseline pattern. One has to keep in mind that, these events are artificially defined and may be manipulated to activate the knee lock at a certain angle. Thus, they do not necessarily reflect actual heel-strike or toe-off events within the gait cycle. Using four sets of pressure sensors manifests itself as a significant advantage in this control algorithm since it enables the therapist to switch between pressure sensor sets for event detection. For instance, either Pressure Sensor Set 1 or Pressure Sensor Set 2 can be used for heel-strike detection while the user has the freedom to choose between Pressure Sensor Set 3 and Pressure Sensor Set 4 for toe-off detection.

To begin with, a threshold value is determined for the pressure sensors within the software in order to define heel-strike and toe-off events. Once a pressure sensor reading exceeds or falls below the threshold value, events can be identified. For instance, the rising edge of Pressure Sensor Set 2 is used in order to define the heel strike event as shown in Figure 3.14. In other words, as soon as Pressure Sensor Set 2 reading exceeds the threshold value, which is defined as 25 lbs in this specific case, the heel-strike event occurs. Falling edge of Pressure Sensor Set 3 is used for toe-off detection. Therefore, the toe-off event occurs when the pressure sensor reading falls below the threshold. During experiments, generally different threshold values are selected for heel-strike and toe-off detection because subjects have a tendency to apply more force at the heel-strike event than the toe-off event. For this reason, a higher threshold value is selected for heel-strike detection.

Once the gait events are detected, lock angle is selected considering existing 


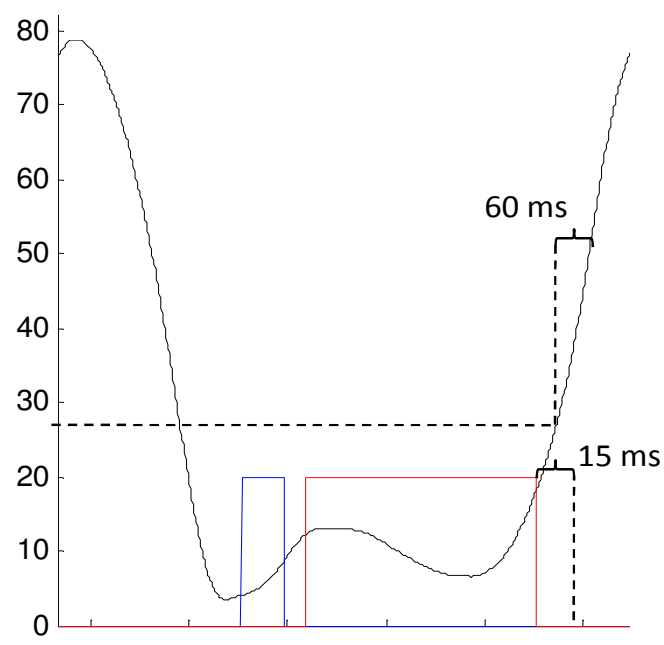

Figure 3.14: Baseline data for the right knee angle (black), pressure sensor set 2 (blue) and set 3 (red) are shown. Selected lock angle must occur at least $15 \mathrm{~ms}$ later than gait events and the lock can be engaged $60 \mathrm{~ms}$ after the magnetic encoder reads this angle.

time delays within the system. As illustrated in Figure 3.14, while selecting an appropriate lock angle, $15 \mathrm{~ms}$ of time delay in response to pressure sensor data and $60 \mathrm{~ms}$ of time delay in response to magnetic encoder data must be taken into account. For this reason, the selected lock angle must occur at least $15 \mathrm{~ms}$ later than the falling edge of the red curve. Furthermore, the lock can be engaged $60 \mathrm{~ms}$ after the selected angle. As a result, if the actual joint angle is desired to be locked at $50^{\circ}$ of flexion, the lock angle must be set to $27^{\circ}$ in the software in order to ensure a secure distance from the time delays for this specific subject.

As shown in Figure 3.6, maximum flexion angle can reach over $80^{\circ}$ for this subject but the flexion lock cannot be engaged at these values due to the design of the gear mechanism. Generally, the maximum knee flexion angle at which the lock could be engaged is $55^{\circ}$ but this value is subject to change. There may be an offset in the brace angle due to the fit of the brace on the subject and this offset may differ from one subject to another. Under these circumstances, engaging 
the flexion lock at $55^{\circ}$ may be feasible for a certain subject while it may not for another. Therefore, flexion lock angle in early swing phase is lower-bounded by the existent time delays and upper-bounded by the gear mechanism. On the other hand, the span of the lock angle can be extended by switching between pressure sensor sets, by switching between rising edges and falling edges of the signals for event detection, or by manipulating threshold values. For instance, using the falling edge of Pressure Sensor Set 3 instead of the rising edge of Pressure Sensor Set 4 in this specific example increases the range of attainable flexion angles considerably. Selecting a higher threshold value narrows the event duration whereas a smaller threshold value widens the duration. Thus, a higher threshold value for Pressure Sensor Set 3 may be selected in order to expand the span of flexion lock angles. After careful inspection of the baseline pattern along with necessary manipulations, the flexion lock is engaged at $40^{\circ}$ as shown in Figure 3.6.

The lock is engaged after the toe-off of the right leg and $60 \mathrm{~ms}$ after the lock angle, which is $25^{\circ}$. The actual joint angle at the instant of lock engagement is approximately $40^{\circ}$, as desired. As shown in Figure 3.6, the engagement of the lock at $37^{\circ}$ instead of $40^{\circ}$ can be explained by two main reasons; (i) offset in the knee joint angle due to the fit of the brace on the subject, and (ii) the gear resolution. The former is discussed in detail in Section 3.1. Gear resolution has a crucial effect on the lock angle. Each gear tooth is seperated by $5^{\circ}$. If the offset value can be set to $0^{\circ}$, the knee joint can be locked at degrees that are multiples of 5 . However, setting the offset to $0^{\circ}$ may not be feasible for each subject, and hence the actual joint angle at the instant of lock engagement can be $37^{\circ}$ instead of $40^{\circ}$ as shown in Figure 3.6.

Furthermore, the duration to keep the lock engaged can also be set within the software. In this specific trial, it takes approximately $200 \mathrm{~ms}$ from the actual lock angle to the point where the subject starts extending the knee. However, 
investigation of experimental data reveals that most of the subjects have a tendency to extend their knees as soon as they feel the perturbation by the flexion lock. Once the subject moves the leg by $2^{\circ}$ in the opposite direction of the lock, the lock disengages. For this reason, the actual lock duration takes less than the value set within the software.

\subsubsection{Extension Lock in Late Swing Phase}

In this strategy, the knee lock is activated when the subject extends the knee during the late-swing phase. As seen in Figure 3.15, subject's left leg is on the ground and the right leg swings forward during the late-swing phase. The lock gets engaged before the heel-strike of the right leg and it is kept locked until the heel strike of the right leg.

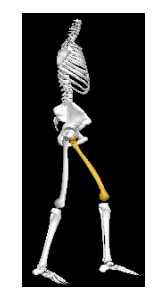

Figure 3.15: A subject in the late swing phase.

Similar to the flexion lock in early-swing phase, baseline gait pattern of the subject must be inspected carefully for appropriate lock settings. First of all, the lock angle is selected considering the existing time delays within the system. For instance, $20^{\circ}$ of extension is aimed to engage the lock for this specific subject. Considering the time delay in response to the magnetic encoder reading, the lock angle needs to be set as $40^{\circ}$ within the software; however, other limitations of the system must also be taken into consideration before setting the lock angle.

Heel-strike and toe-off events have to be defined clearly on the baseline. One has to make sure that the pressure sensor data are strong enough before selecting threshold values for heel-strike and toe-off events. Heel-strike event has a crucial role 
in this lock strategy since the lock must be engaged in the swing phase. Heel-strike event defines the beginning of the stance phase. As soon as the subject gets into the stance phase the lock disengages. Using the rising edge of Pressure Sensor Set 2 instead of the rising edge of Pressure Sensor Set 1, can increase the duration to keep the lock engaged. This duration may further be increased by selecting a higher threshold value for the heel-strike event while this increase is limited by the strength of the pressure sensor signal. Moreover, the software is improved to allow the user to define the events at the end of a desired amount of time. As a result, this feature of the program introduces an extra time to keep the lock engaged when necessary. In addition to the heel-strike event, two more conditions are available to ensure the disengagement of the lock before the subject gets into the stance phase. First one is the adjustable lock duration. Inspecting the baseline pattern of the subject enables the user to calculate the duration to keep the lock engaged while the subject is extending the knee in late-swing phase. The lock gets disengaged at the end of this specified period. As shown in Figure 3.7, the subject takes on average $200 \mathrm{~ms}$ to start flexing the knee from the desired lock angle. Therefore, the lock duration can be set as $200 \mathrm{~ms}$, and at the end of this period a signal is sent to disengage the lock. The final condition that guarantees the disengagement of the lock during the stance phase is flexing the knee by $2^{\circ}$.

Apart from the heel-strike event, definition of the toe-off event has a major role in the lock engagement. In order to prevent the lock from being activated while the subject is flexing the knee in the early swing phase, the selected angle must occur at most $15 \mathrm{~ms}$ later than the toe-off event due to the time delay in response to the pressure sensor reading. The extension lock engages if the selected lock angle is smaller than the knee angle, which is read by the magnetic encoder. Consequently, the angle value, which corresponds to $15 \mathrm{~ms}$ after the falling edge of Pressure Sensor Set 4 , must be found and if this value is smaller than the selected lock angle, a new 
lock angle must be selected or the event definition must be manipulated so as to engage the lock at that specific angle. The gear mechanism does not allow the user to engage the extension lock while the subject is flexing the knee and by the same token the flexion lock cannot be engaged while the subject is extending the knee.

After careful inspection of the baseline gait pattern along with necessary manipulations, the extension lock is engaged during the late-swing phase at $20^{\circ}$ as shown in Figure 3.7.

As observed in Figure 3.7, the lock is engaged $60 \mathrm{~ms}$ after the magnetic encoder reading indicates a smaller value $\left(39^{\circ}\right)$ than the lock angle $\left(40^{\circ}\right)$. Taking the limitations of the device such as time delays and the design of the gear mechanism into account resulted in a reliable lock trial. However, engagement of the extension lock during late-swing phase has a number of challenges.

First, the treadmill speed has a significant effect on the actual lock angle. The difference between the actual joint angle and the lock angle shows a variation for different treadmill speeds owing to the time delays. In order to overcome this problem, the treadmill speed is fixed at 1 meter/second throughout experiments and the subject's comfort is ensured with the speed.

Besides the treadmill speed, the knee flexion during the stance phase is a significant point to consider for the settings of the extension lock in the late-swing phase. In order for the lock to get disengaged in the stance phase, the subject must flex the knee at least by $2^{o}$. Even though the signal to disengage the lock is sent, the lock cannot be released unless there is $2^{\circ}$ of flexion. On the other hand, some of the subjects do not tend to flex their knees during the stance phase. Figure 3.16 shows baseline patterns of the right knee angle for two different subjects. As can be seen in Figure 3.16 (b), the subject does not flex the knee during the stance phase. Therefore, the lock cannot be disengaged once it is engaged in the late-swing phase.

Finally, a single stride in the baseline is taken into account while calculating 


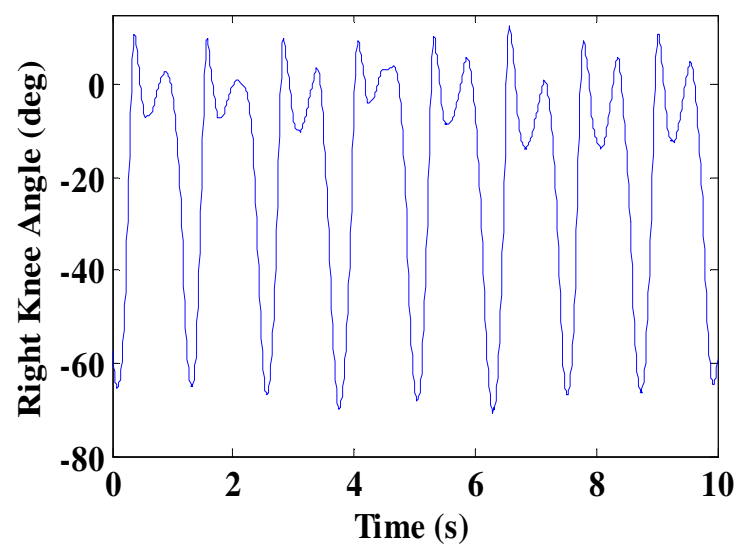

a)

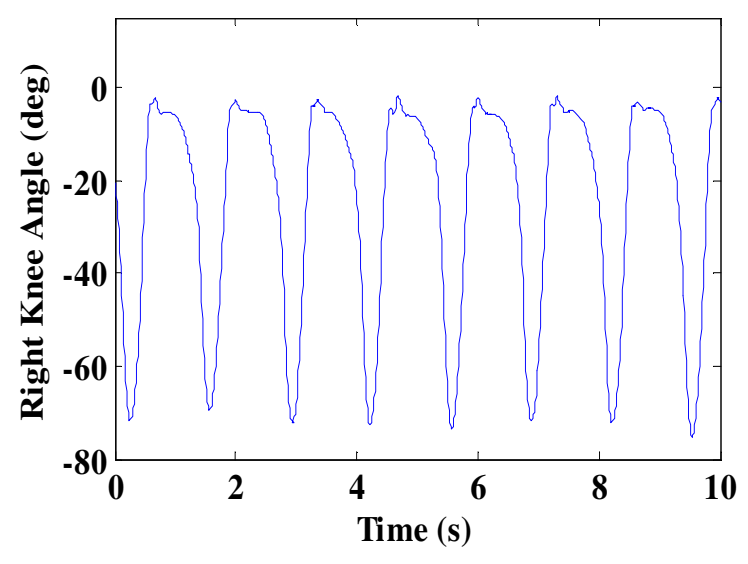

b)

Figure 3.16: Baseline right knee angle for (a) subject 6 and (b) subject 4 . Subject 4 does not flex the knee during the stance phase so the extension lock cannot be disengaged once it is engaged in the late swing phase.

the angle values and lock durations. In each stride there are minor variations in durations and angle values. Thus, the lock may engage at a different angle than the expected value.

\subsubsection{Flexion Lock in Stance Phase}

The knee lock is activated when the subject flexes the right knee during the stance phase. As seen in Figure 3.17, subject's right leg is on the ground and the left leg swings backwards in this phase. The lock is engaged following the heel-strike of the right leg and it is kept locked until the subject starts extending the knee.

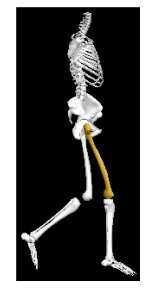

Figure 3.17: A subject in stance phase. 
After manipulating threshold values and switching between pressure sensor sets for event detection, lock angle is selected as $25^{\circ}$ for this specific subject, Figure 3.18 .

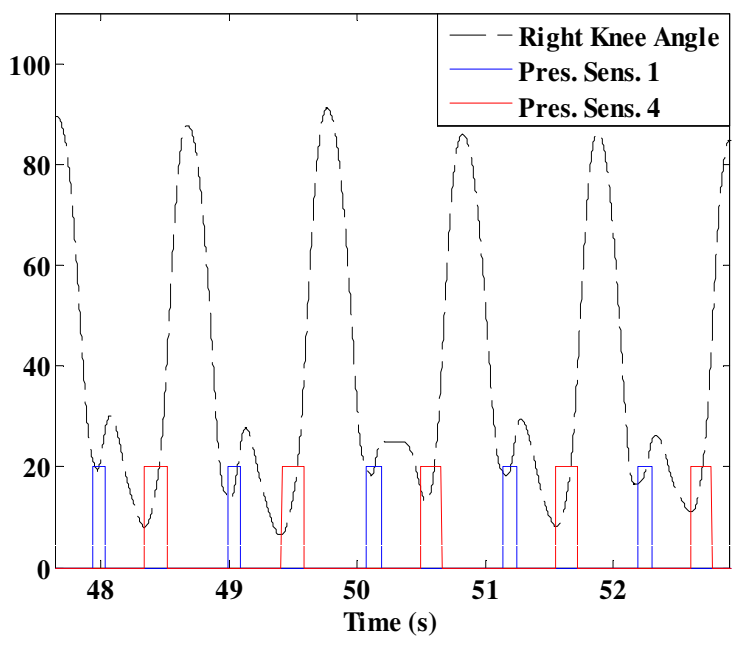

Figure 3.18: Flexion lock in the stance phase induced by SKB. Right knee angle (dashed), pressure sensor set 1 (blue) and set 4 (red) are shown.

Although the lock duration is set as $300 \mathrm{~ms}$, the subject starts extending the knee sooner than $300 \mathrm{~ms}$. Once the subject extends the knee by $2^{o}$, the lock gets disengaged.

\subsubsection{Extension Lock in Stance Phase}

In this strategy, the knee lock is activated when the subject extends the knee during the mid-stance phase. As seen in Figure 3.19, subjects right leg is on the ground and the left leg swings forward during the mid-stance phase. The lock gets engaged following the heel-strike of the right leg and it is kept locked until the toe-off of the right leg.

Heel-strike detection has an essential role in this lock strategy given that the lock is engaged right after the heel-strike. As discussed in the previous lock strategies, manipulation of threshold values and switching between pressure sensor 


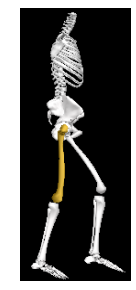

Figure 3.19: A subject in stance phase.

sets are realized for event definition when necessary. After defining the heel-strike event, the lock angle needs to be selected. For instance, analyzing the baseline gait pattern reveals that $20^{\circ}$ of extension is reasonable to engage the lock for this specific subject, as depicted in Figure 3.20. Considering the time delay in response to the pressure sensor and magnetic encoder data, lock angle needs to be set as $30^{\circ}$ within the software. The subject takes on average $450 \mathrm{~ms}$ from the heel-strike to start flexing the knee. Therefore, the lock duration is set as $450 \mathrm{~ms}$ and the falling edge of Pressure Sensor Set 4 is used for toe-off detection. After careful inspection of the baseline gait pattern along with necessary manipulations, the extension lock is engaged during the mid-stance phase at $20^{\circ}$, as shown in Figure 3.20.

Even though the lock duration is set as $450 \mathrm{~ms}$ within the software, the actual duration during the experiment may be different than this value due to three conditions. First, the duration is measured based on a single stride on the baseline and may show slight variations from one stride to another. Second, the toe-off event releases the lock and hence the lock will be disengaged following the toe-off event regardless of the lock duration. Third, flexing the knee by $2^{o}$ unlocks the knee joint in spite of the lock duration. Under these circumstances, the actual lock duration may be different than the value set within the software. 


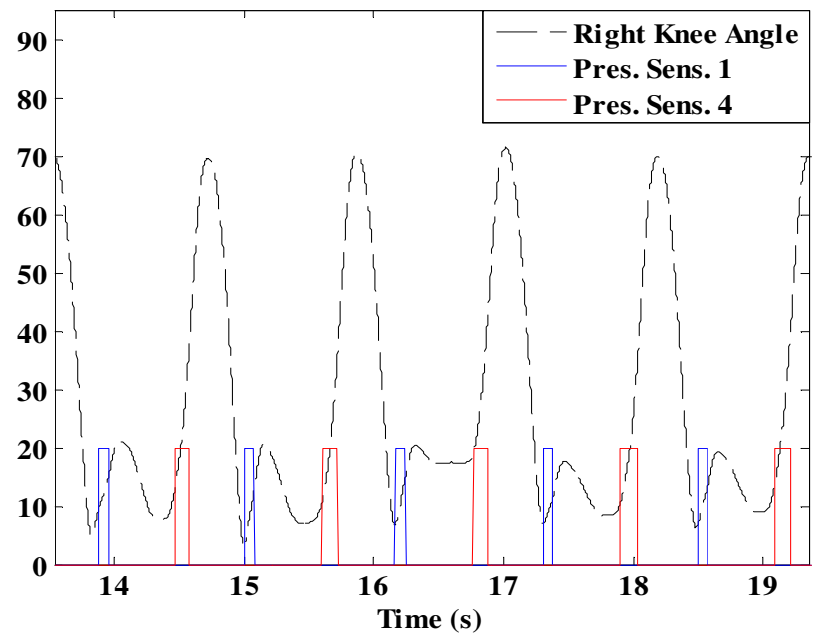

Figure 3.20: Extension lock in the stance phase induced by SKB. Right knee angle (dashed), pressure sensor set 1 (blue) and set 4 (red) are shown. 


\section{Chapter 4}

\section{GAIT CHARACTERIZATION}

Controlled perturbations applied via the SKB are used to quantify the response of different groups of subjects. To evaluate the human applied joint torques to hypothesize how the human neuro-muscular system modulates the joint torques as a response to the perturbations caused on the gait, a dynamic model of human walking is created. Using this model, the neuro-muscular response to perturbations can be reasonably well characterized by including the following features in the model: (i) normal gait in the absence of perturbation, (ii) corrective torque at a joint in response to the error at that joint and other joints, (iii) a characteristic time shift in the response. These parameters are then used to quantify the behavior of subjects under controlled kinematic perturbations to one limb during gait.

\subsection{Dynamic Model of Human Walking During Swing Phase}

In order to quantify the kinetics of swing, a four degree-of-freedom human walking model was developed. This model does not account for the double support phase. Rather, it assumes that the heel of the stance foot is in contact with the ground independently. Each leg moves in the sagittal plane and consists of a hip and knee joint. The hip and knee segments have distributed mass, based on anthropometric data [3], and a point mass at the hip represents the contributions of the upper trunk. This is shown schematically in Figure 4.1, where the biped changes its stance from the left leg to the right leg. 


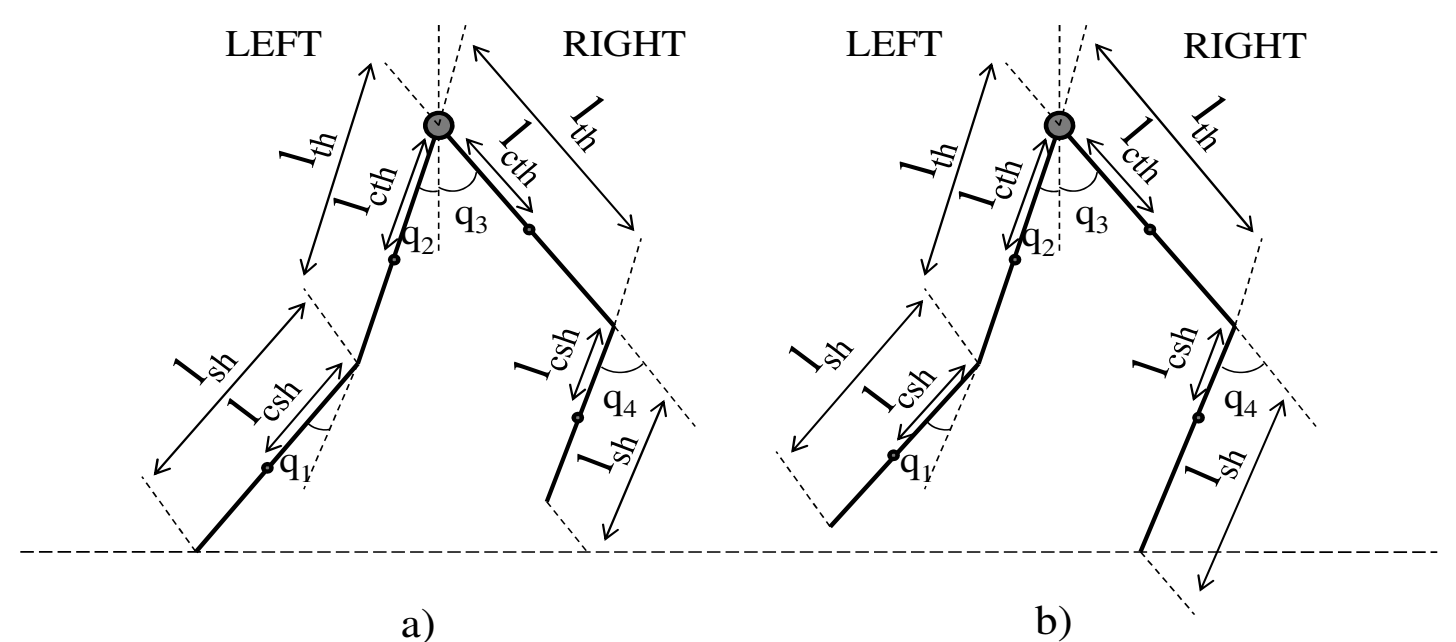

Figure 4.1: A four degree-of-freedom sagittal plane biped model of human standing on left leg and right leg. The model assumes that the heel of the stance leg remains in contact with the ground until the heel strike of the other leg. The hip and knee segments have distributed mass and the trunk is represented by a point mass at the hip joint.

The location of the center of mass for each segment is measured from the proximal end. Mass, inertia and length of each limb are expressed as a percentage of the total body mass or height. Clockwise direction is chosen as the positive direction in the model for joint angles. As shown in Figure 4.1, lsh is the length of the shank segment, lcsh is the location of the center of mass of this segment from the knee joint, lth is the length of the thigh segment and lcth is the location of the center of mass of this segment from the hip joint.

The joint angles are shown in Figure 4.1, $q_{1}$ for the left knee angle, $q_{2}$ for the left hip, $q_{3}$ for the right hip, and $q_{4}$ for the right knee. The model assumes the subject to be in single support phase. The stance leg rolls on the ground without sliding and the subject's motion is assumed to be in the sagittal plane. The walking dynamic model is obtained using the Lagrangian formulation and these equations of motion have the following form: 


$$
D(q) \ddot{q}+C(q, \dot{q}) \dot{q}+G(q)=u
$$

where $q=\left(q_{1}, q_{2}, q_{3}, q_{4}\right)^{T}, D(q)$ is the $(4 \times 4)$ positive definite inertia matrix, $C(q, \dot{q})$ is the matrix of centrifugal and Coriolis terms, $G(q)$ is the gravity matrix and $u=\left(u_{1}, u_{2}, u_{3}, u_{4}\right)$ is the vector of joint torques for hip and knee. In order to calculate joint torques, as a human switches stance from the right leg to the left leg, two different models were developed compatible with sketches, as illustrated in Figure 4.1. The equations of motion are discussed in further detail in Section 4.1.1.

Gait events are detected using the sensors mounted on the SKB and the motion capture system. Motion capture system provides data for joint kinematics during the unlocked condition and after perturbations caused by knee lock in early swing. Using these kinematic data, the dynamic model predicts torques applied at the joints during free knee movement condition and after the locked stride.

\subsubsection{Equations of Motion}

In order to obtain the torques applied by the human at hip and knee joints, the Lagrangian is calculated [3]. The Lagrangian is defined as:

$$
L=K E-P E
$$

where $K E$ is the total kinetic energy and $P E$ is the total potential energy of the system. The potential and kinetic energy of each segment are calculated according to the sketches illustrated in Figure 4.1. The following equations apply when the subject stands on the left leg. As the human switches stance from the left to the right leg, a separate set of equations compatible with Figure 4.1 (b) are derived.

The potential energy of the shank segment of the stance leg is:

$$
P E_{1}=m_{1} * g * h_{1}(t)
$$




$$
\begin{aligned}
m_{1} & =0.0465 * M \\
h_{1}(t) & =(l s h-l c s h) * \cos \left(q_{1}(t)+q_{2}(t)\right) \\
l s h & =0.246 * H \\
l c s h & =0.433 * l s h
\end{aligned}
$$

The potential energy of the thigh segment of the stance leg is:

$$
\begin{aligned}
P E_{2} & =m_{2} * g * h_{2}(t) \\
m_{2} & =0.1 * M \\
h_{2}(t) & =l s h * \cos \left(q_{1}(t)+q_{2}(t)\right)+(l t h-l c t h) * \cos \left(q_{2}(t)\right) \\
l t h & =0.284 * H \\
l c t h & =0.433 * l t h
\end{aligned}
$$

The potential energy of the thigh segment of the swing leg is:

$$
\begin{aligned}
P E_{3} & =m_{2} * g * h_{3}(t) \\
h_{3}(t) & =l s h * \cos \left(q_{1}(t)+q_{2}(t)\right)+l t h * \cos \left(q_{2}(t)\right)-l c t h * \cos \left(q_{3}(t)\right)
\end{aligned}
$$

The potential energy of the shank segment of the swing leg is:

$$
\begin{aligned}
P E_{4}= & m_{1} * g * h_{4}(t) \\
h_{4}(t)= & l s h * \cos \left(q_{1}(t)+q_{2}(t)\right)+l t h * \cos \left(q_{2}(t)\right)-l t h * \cos \left(q_{3}(t)\right) \\
& -l \operatorname{csh} * \cos \left(q_{4}(t)-q_{3}(t)\right)
\end{aligned}
$$

The model assumes the upper body as a point mass at the hip joint. The potential energy of the trunk is:

$$
\begin{aligned}
P E_{5} & =m_{5} * g * h_{5}(t) \\
m_{5} & =0.678 * M \\
h_{5}(t) & =l s h * \cos \left(q_{1}(t)+q_{2}(t)\right)+l t h * \cos \left(q_{2}(t)\right)
\end{aligned}
$$


The kinetic energy of the shank and the thigh segments have linear and rotational components, $v(t)$ and $\omega(t)$ respectively. The kinetic energy of the shank segment of the stance leg is derived as:

$$
\begin{aligned}
K E_{1} & =\frac{1}{2} * m_{1} * v_{1}^{2}(t)+\frac{1}{2} * I_{1} * \omega_{1}^{2}(t) \\
v_{1}(t) & =\operatorname{diff}\left[(l s h-l c s h) *\left(\cos \left(q_{1}(t)+q_{2}(t)\right)+\sin \left(q_{1}(t)+q_{2}(t)\right)\right)\right] \\
\omega_{1}(t) & =\operatorname{diff}\left(q_{1}(t)+q_{2}(t)\right) \\
I_{1} & =m_{1} *(0.302 * l s h)^{2}
\end{aligned}
$$

The kinetic energy of the thigh segment of the stance leg is:

$$
\begin{aligned}
K E_{2}= & \frac{1}{2} * m_{2} * v_{2}^{2}(t)+\frac{1}{2} * I_{2} * \omega_{2}^{2}(t) \\
v_{2}(t)= & \operatorname{diff}\left[l s h *\left(\cos \left(q_{1}(t)+q_{2}(t)\right)+\sin \left(q_{1}(t)+q_{2}(t)\right)\right)\right. \\
& \left.+(l t h-l c t h) *\left(\cos \left(q_{2}(t)\right)+\sin \left(q_{2}(t)\right)\right)\right] \\
\omega_{2}(t)= & \operatorname{diff}\left(q_{2}(t)\right) \\
I_{2}= & m_{2} *(0.323 * l t h)^{2}
\end{aligned}
$$

The kinetic energy of the thigh segment of the swing leg is:

$$
\begin{aligned}
K E_{3}= & \frac{1}{2} * m_{2} * v_{3}^{2}(t)+\frac{1}{2} * I_{2} * \omega_{3}^{2}(t) \\
v_{3}(t)= & \operatorname{diff}\left[l \operatorname{sh} *\left(\cos \left(q_{1}(t)+q_{2}(t)\right)+\sin \left(q_{1}(t)+q_{2}(t)\right)\right)\right. \\
& \left.+\operatorname{lth} *\left(\cos \left(q_{2}(t)\right)+\sin \left(q_{2}(t)\right)\right)+l c t h *\left(\sin \left(q_{3}(t)\right)-\cos \left(q_{3}(t)\right)\right)\right] \\
\omega_{3}(t)= & \operatorname{diff}\left(q_{3}(t)\right)
\end{aligned}
$$

The kinetic energy of the shank segment of the swing leg is:

$$
\begin{aligned}
K E_{4}= & \frac{1}{2} * m_{1} * v_{4}^{2}(t)+\frac{1}{2} * I_{1} * \omega_{4}^{2}(t) \\
v_{4}(t)= & \operatorname{diff}\left[l s h *\left(\cos \left(q_{1}(t)+q_{2}(t)\right)+\sin \left(q_{1}(t)+q_{2}(t)\right)\right)\right. \\
& +l t h *\left(\cos \left(q_{2}(t)\right)+\sin \left(q_{2}(t)\right)\right)+l t h *\left(\sin \left(q_{3}(t)\right)-\cos \left(q_{3}(t)\right)\right) \\
& \left.+l \operatorname{csh} *\left(\sin \left(q_{4}(t)-q_{3}(t)\right)-\cos \left(q_{4}(t)-q_{3}(t)\right)\right)\right] \\
\omega_{4}(t)= & \operatorname{diff}\left(q_{4}(t)-q_{3}(t)\right)
\end{aligned}
$$


The kinetic energy of the trunk includes only the linear component since the trunk is assumed to be a point mass at the hip joint and it is derived as:

$$
\begin{aligned}
K E_{5}= & \frac{1}{2} * m_{5} * v_{5}^{2}(t) \\
v_{5}(t)= & \operatorname{diff}\left[\operatorname{lsh} *\left(\cos \left(q_{1}(t)+q_{2}(t)\right)+\sin \left(q_{1}(t)+q_{2}(t)\right)\right)\right. \\
& \left.+l t h *\left(\cos \left(q_{2}(t)\right)+\sin \left(q_{2}(t)\right)\right)\right]
\end{aligned}
$$

where $M$ is the total body mass and $H$ is the total body height. Mass $\left(m_{i}\right), i=$

$1, . ., 5$, inertia $\left(I_{j}\right), j=1,2$ and length of each segment are expressed as a percentage of the total body mass and height. Motion capture system provides the kinematical data. Once the joint kinematics are known, the Lagrangian of the four degree-offreedom human walking model can be calculated using Equations (4.2) to (4.12). Hip and knee joint torques are derived from the Lagrangian of the system using Equation (4.13).

$$
\frac{d}{d t} \frac{\partial L}{\partial \dot{q}_{j}}-\frac{\partial L}{\partial q_{j}}=\tau_{j}
$$

where $q_{j}, j=1, \ldots, 4$ represents the joint position, $\dot{q}_{j}, j=1, . ., 4$ is the joint velocity and $\tau_{j}$ is the corresponding joint torque. Therefore, a joint torque is calculated by the corresponding joint position and velocity in Equation (4.13). Hip and knee joint torques are calculated by using the kinematical data during the unlocked condition and after perturbations caused by knee lock in early swing.

\subsection{Human Neuro-muscular Response}

Human neuro-muscular system responds to perturbations during gait and by attempting to restore the normal gait kinematics by modulating the torques applied at the joints. Each subject responds to perturbations differently but all healthy 
subjects restore their normal walking pattern. The characterization of the neuromuscular response of individuals to perturbations, i.e., how an individual modulates joint torques may provide insight into who is more prone to falls.

The neuro-muscular response is characterized by different control laws: (i) independent joint control - error from the normal motion at a joint is used to modulate the torques applied at that joint and, (ii) coordinated joint control - errors from the normal motion of all joints are used to modulate the torque input at one joint. These control laws alone are not enough to explain the human applied joint torques after the perturbation. These control laws need to include a characteristic time shift, as described in the following sections.

\subsubsection{Independent Joint Control}

The neuro-muscular response to a perturbation in gait is modeled by two terms: (i) a model of the normal motion, (ii) a proportional-derivative controller that attempts to annul the error between the perturbed motion and the normal motion. Mathematically, this is described as

$$
u_{i}(t)=u_{n i}(t)+K_{p i}\left(q_{n i}(t)-q_{i}(t)\right)+K_{d i}\left(\dot{q}_{n i}(t)-\dot{q}_{i}(t)\right)
$$

where $u_{i}(t), i=1, . ., 4$, represents the torque at joint $i$ after the perturbation, $u_{n i}(t)$ is the normal joint torque, $K_{p i}$ is the proportional gain constant and $K_{d i}$ is the derivative gain constant. The normal kinematic data, $q_{n i}(t), \dot{q}_{n i}(t)$, and the perturbed kinematic data $q_{i}(t)$ and $\dot{q}_{i}(t)$ are obtained via the camera system. Joint torques, $u_{i}(t)$ and $u_{n i}(t)$, are calculated using the dynamic model.

The parameters $K_{p i}$ and $K_{d i}$ are obtained by least squares routine from Matlab. The algorithm seeks a parameter vector that minimizes the norm of the vector $\left\|u_{i}-u_{n i}-K_{p i}\left(q_{n i}-q_{i}\right)-K_{d i}\left(\dot{q}_{n i}-\dot{q}_{i}\right)\right\|$. The rank of the matrix below, containing 
the kinematic error terms, is determined from the QR decomposition with column pivoting. Equation (4.14) is written in the matrix form given by Equation (4.15).

$$
\left[\begin{array}{c}
u_{i j}-u_{n i j} \\
\cdot \\
\cdot \\
\cdot
\end{array}\right]=\left[\begin{array}{cc}
q_{n i j}-q_{i j} & \dot{q}_{n i j}-\dot{q}_{i j} \\
\cdot & \cdot \\
\cdot & \cdot \\
\cdot & \cdot \\
\cdot & \cdot
\end{array}\right]\left[\begin{array}{c}
K_{p i} \\
K_{d i}
\end{array}\right]
$$

for $i=1, \ldots, 4$ and $j=1, \ldots, N$, where $N$ is the number of grid points. Figure 4.2(a) shows a data set of normal and perturbed joint angles for the right knee over one stride after the knee was unlocked. Figure 4.2(b) shows torque generated during unlocked trials and perturbed trials along with the predicted applied torque $u_{i}(t)$ using Equation (4.14) obtained with the best parameters $K_{p i}$ and $K_{d i}$. The figure represents a trial in which the torque values are normalized by the total body mass of the subject. The corresponding joint angle data and the torque data before and after the perturbation along with the predicted torque with best parameters of $K_{p i}$ and $K_{d i}$ for the left knee are shown in Figure 4.2(c) and Figure 4.2(d). Data for the hip joints are shown in Figure 4.3. One can observe from these figures that the model of the neuro-muscular response given by Equation (4.14) does not capture the experiment data well. 


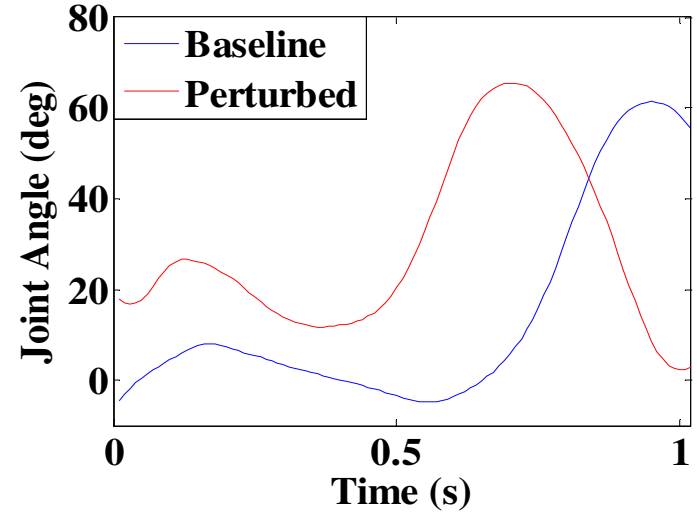

a)

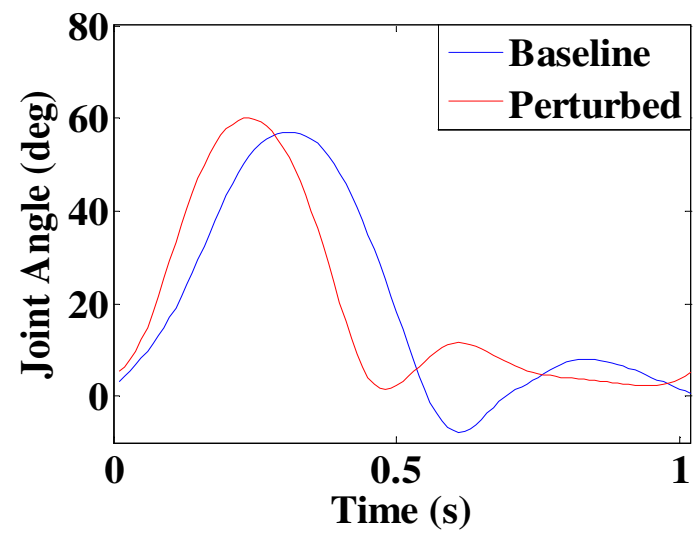

c)

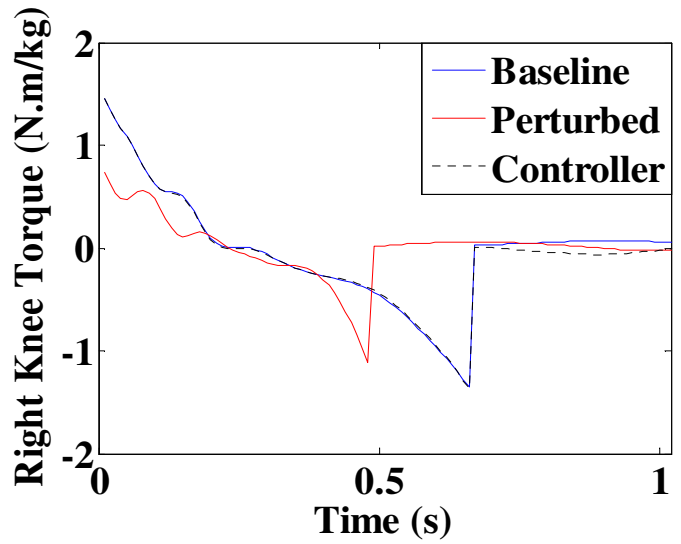

b)

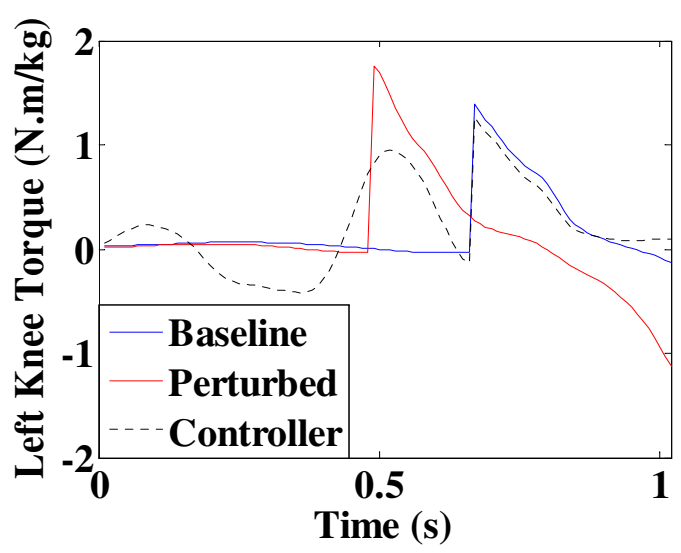

d)

Figure 4.2: Kinematic data (a) and kinetic data (b) for the right knee and similar data for the left knee joints (c), (d). Normal (blue) and perturbed (red) data are shown. Predicted applied torque (dashed) is obtained with the best parameters $K_{p i}$ and $K_{d i}$ of the independent joint control. 


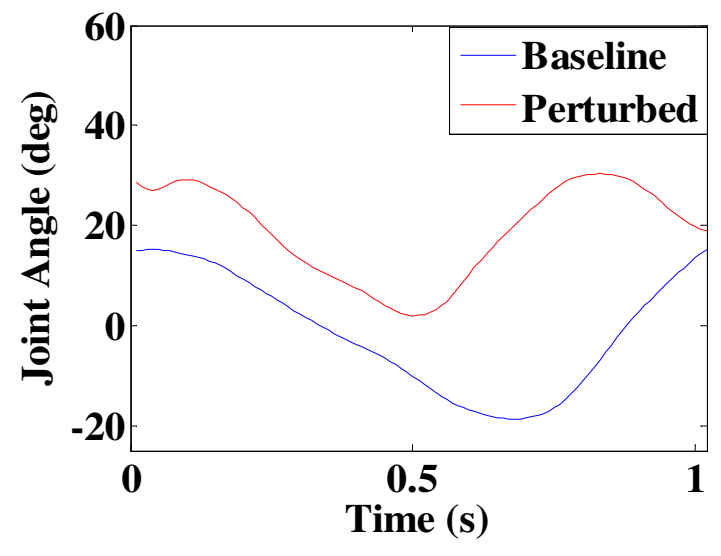

a)

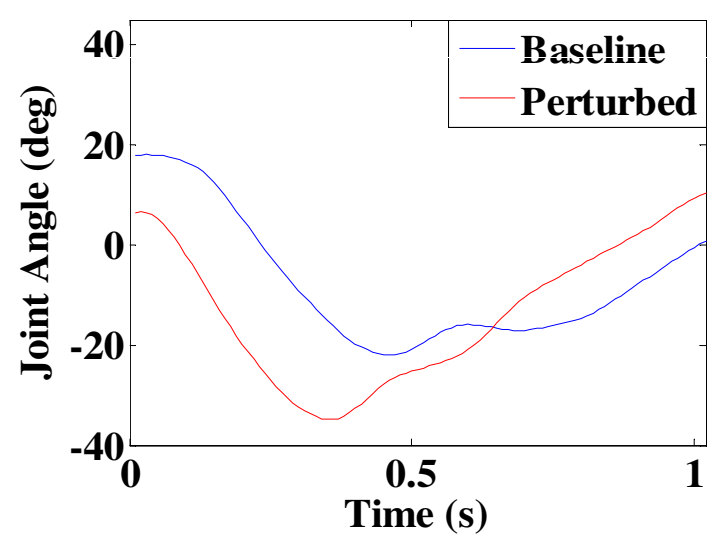

c)

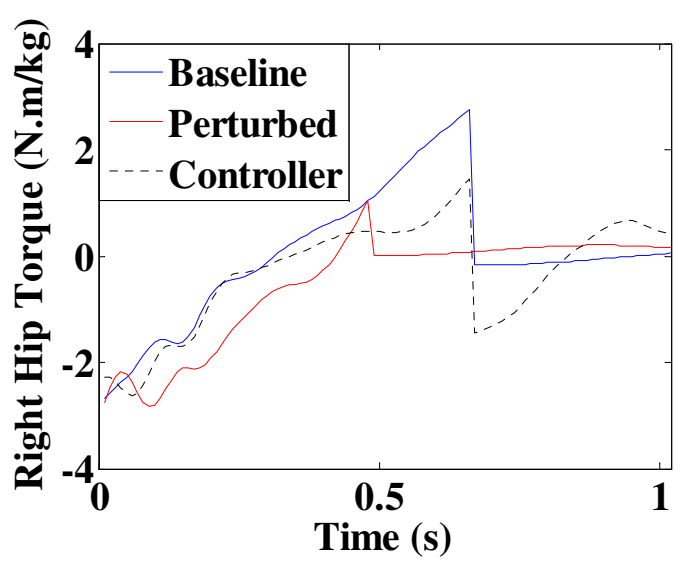

b)

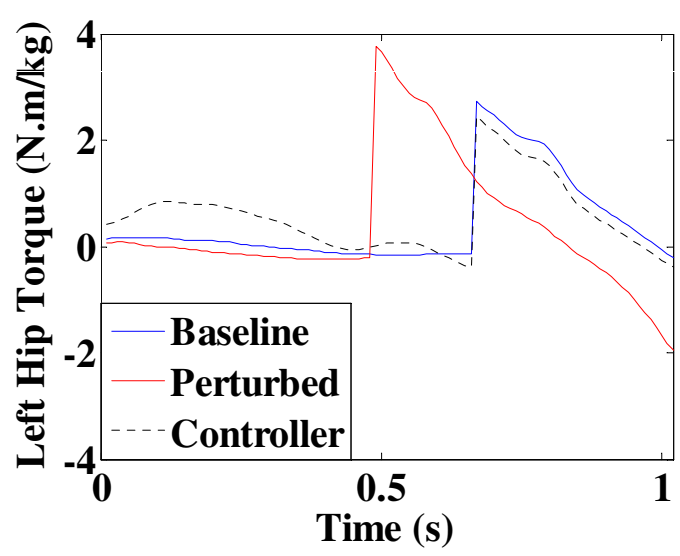

d)

Figure 4.3: Kinematic data (a) and kinetic data (b) for the right hip joint and similar date for the left hip joint (c), (d). Normal (blue) and perturbed (red) data are shown. Predicted applied torque (dashed) is obtained with the best parameters $K_{p i}$ and $K_{d i}$ of the independent joint control. 


\subsubsection{Coordinated Joint Control}

In the coordinated joint model, the torque response at a joint, after the lock is disengaged, is based on errors from the normal motion at all joints and is given by

$$
u_{i}(t)=u_{n i}(t)+\sum_{j=1}^{4} K_{p i j}\left(q_{n j}(t)-q_{j}(t)\right)+\sum_{j=1}^{4} K_{d i j}\left(\dot{q}_{n j}(t)-\dot{q}_{j}(t)\right)
$$

Equation (4.16) can be written in a matrix form as shown in Equation (4.17). The unknown matrix consists of 16 parameters for position errors and another 16 for the velocity errors, in contrast to 4 parameters each in the independent joint controller. Matlab algoritm described in the previous section is used again to find the parameters, $K_{p i}$ and $K_{d i}$ with the least square method.

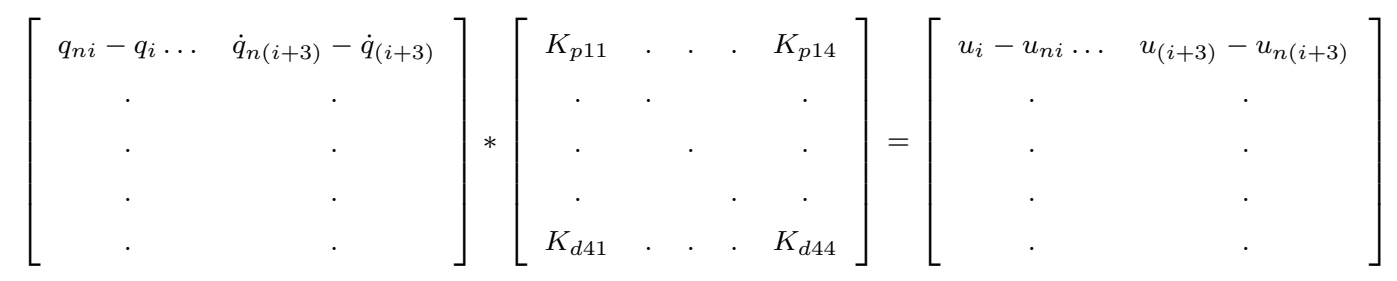

Figure 4.4 shows the controller results for each joint and this coordinated joint control model is more effective in predicting the actual responses compared to the previous controller. 


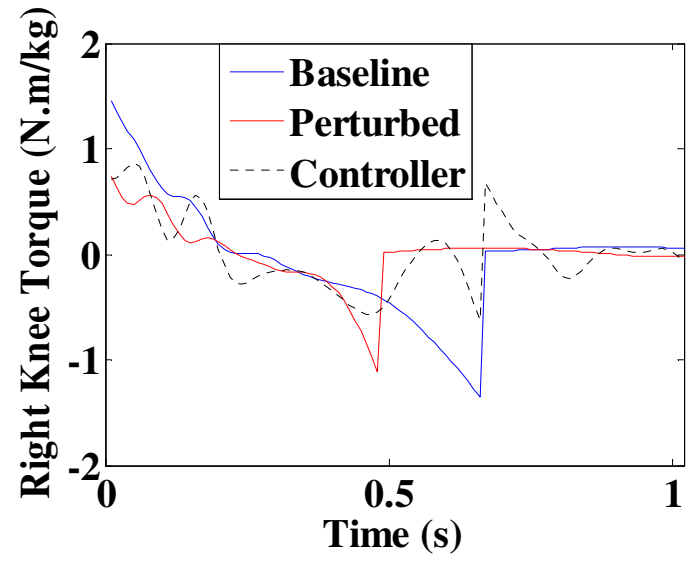

a)

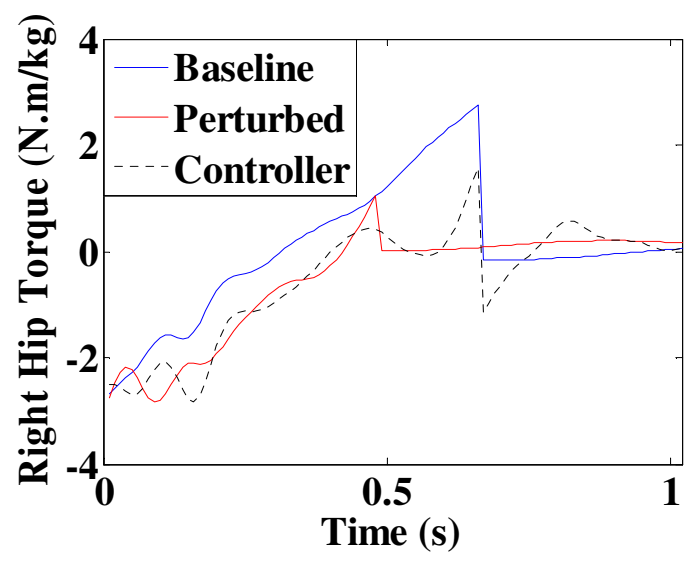

c)

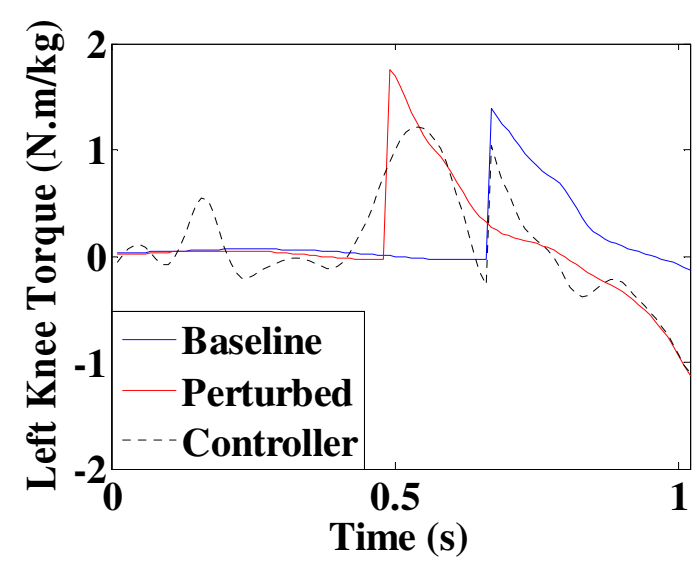

b)

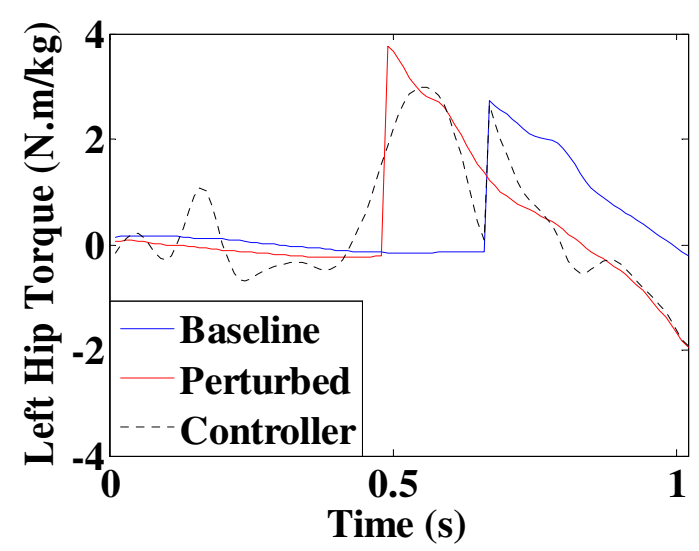

d)

Figure 4.4: Kinetic data for the knee (a), (b) and for the hip joints (c), (d). Normal (blue) and perturbed (red) data are shown. Predicted applied torque (dashed) is obtained with the best parameters $K_{p i}$ and $K_{d i}$ of the coordinated joint control. 


\subsubsection{Independent Joint Control with Time Shift}

It is hypothesized that the sensory information is processed by human neuromuscular system using the following two motor control mechanisms. The first is a feedback mechanism in which sensory information received from the joints and muscles is integrated with other sensory information such as ocular and the vestibular input to control motion. The reflex loops are associated with feedback loops and require a processing time of up to (200ms) which is too long to be of use in responding to unexpected perturbations. On the other hand, an open-loop system is one in which relevant muscles are pre-tuned, so that the pre-activated muscles can react faster ( $30 \mathrm{~ms}-80 \mathrm{~ms}$ ) when a perturbation to movement occurs [2]. Therefore, a time shift must be included in any model of joint control to account for rapid muscle responses to improve the performance of the controller response.

This time shift is introduced into the independent joint control model as shown in Equation (4.18). Intuitively, this relation suggests that the neuro-muscular system responds to the perturbation by generating joint torques more quickly than it would under the unlocked condition.

$$
u_{i}(t)=u_{n i}(t)+K_{p i}\left(q_{n i}(t)-q_{i}\left(t-t_{d}\right)\right)+K_{d i}\left(\dot{q}_{n i}(t)-\dot{q}_{i}\left(t-t_{d}\right)\right)
$$

To determine the optimal time shift, total controller error is minimized using the Matlab routine described in the previous sections within a loop as the duration of the time shift is changed. The very first stride after the locked stride is compared with the data from the unlocked baseline strides in the following figures. Figure 4.5, shows the controller results for each joint. As far as the single joint controller with time shift, the results demonstrate improved tracking responses for the left knee and left hip joints. However, tracking response for the other joints still need to be improved. 


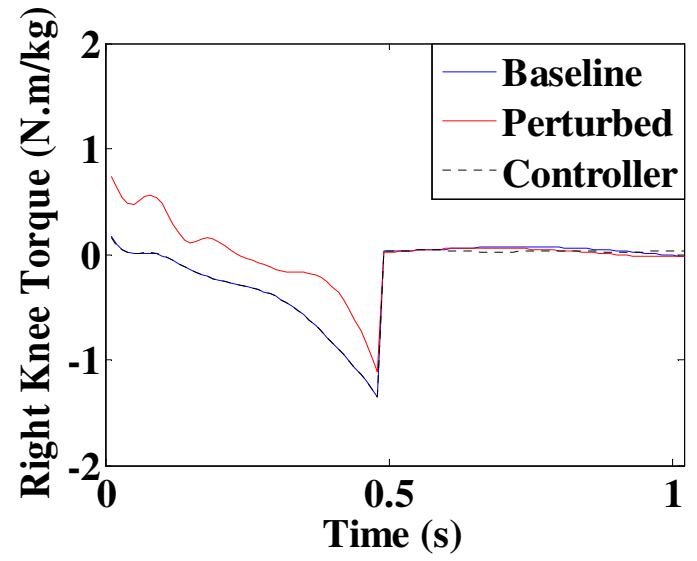

a)

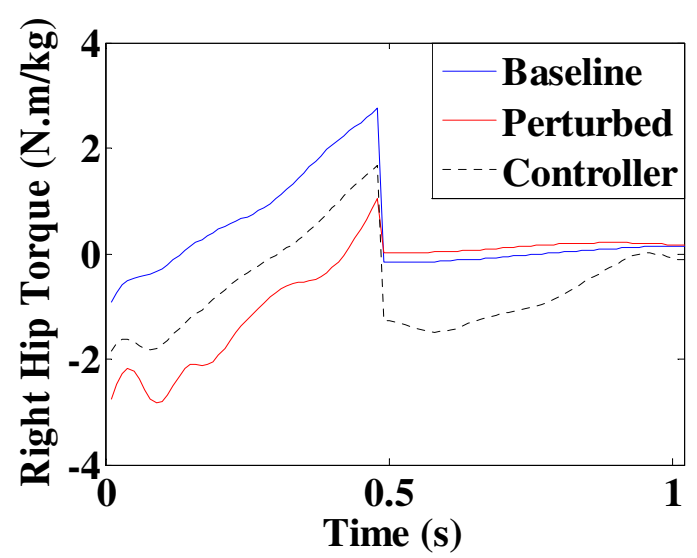

c)

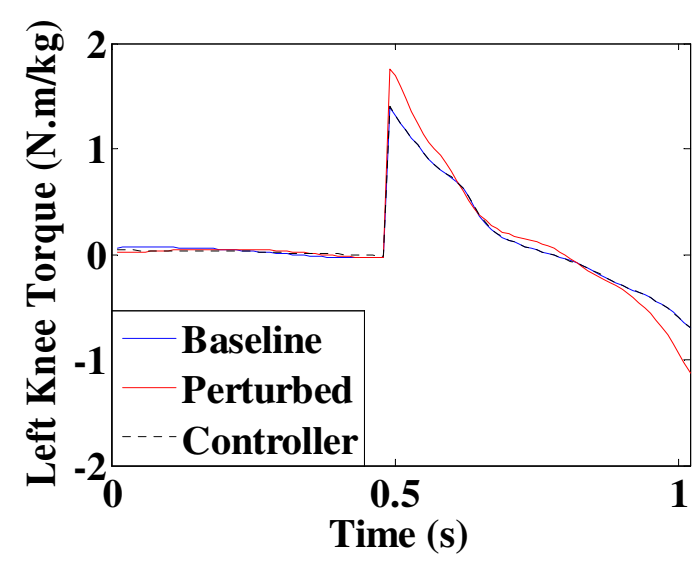

b)

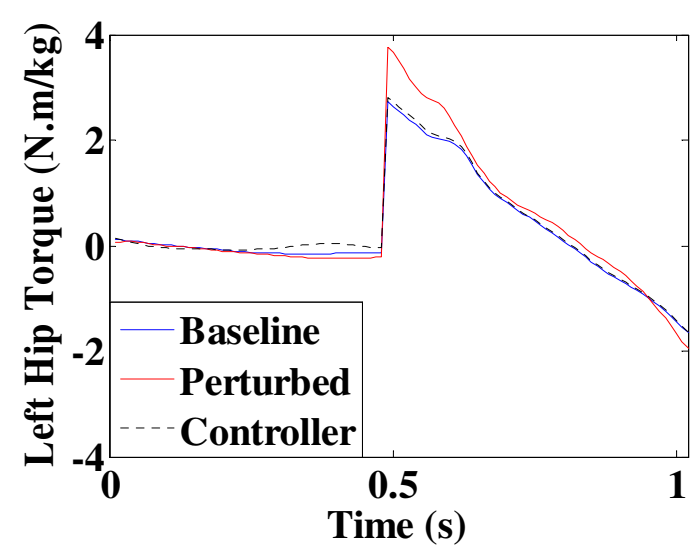

d)

Figure 4.5: Kinetic data for the knee (a), (b) and for the hip joints (c), (d). Normal (blue) and perturbed (red) data are shown. Predicted applied torque (dashed) is obtained with the best parameters $K_{p i}$ and $K_{d i}$ of the independent joint control with time shift. 


\subsubsection{Coordinated Joint Control with Time Shift}

In this model, the torque response at the joints in the stride immediately following the locked stride is based on errors at all of the joints and includes a characteristic time shift and is given by

$$
u_{i}(t)=u_{n i}(t)+\sum_{j=1}^{4} K_{p i j}\left(q_{n j}(t)-q_{j}\left(t-t_{d}\right)\right)+\sum_{j=1}^{4} K_{d i j}\left(\dot{q}_{n j}(t)-\dot{q}_{j}\left(t-t_{d}\right)\right)
$$

Figure 4.6 shows the results of the coordinated joint control model for each

joint. With this corrective neuro-muscular response to a perturbation in the gait, the model provides a reasonable match between the predicted and observed responses.

\subsubsection{Experimental Results}

This section summarizes the experimental protocol and presents a discussion of the parameters calculated by the Coordinated Joint Control with Time Shift. 


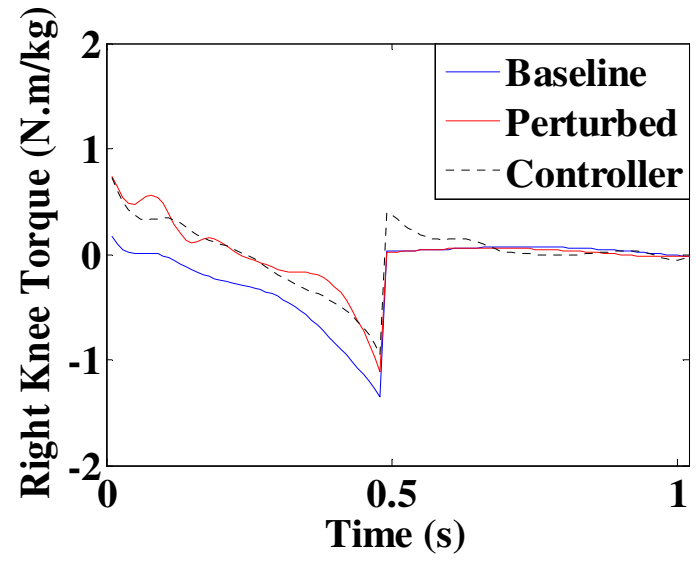

a)

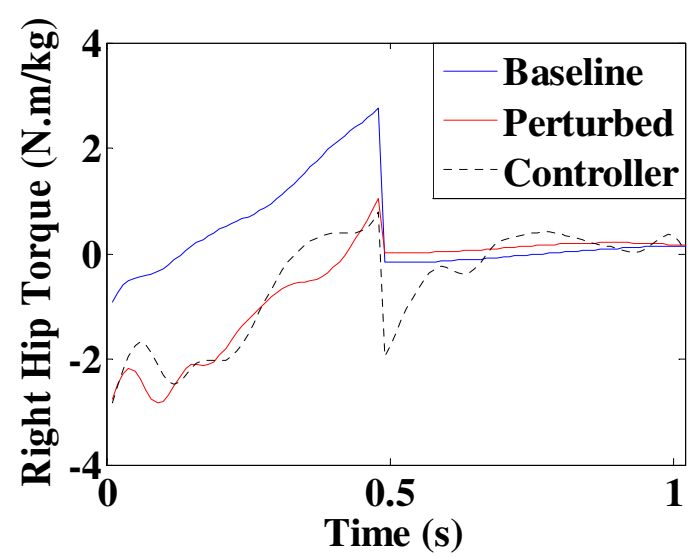

c)

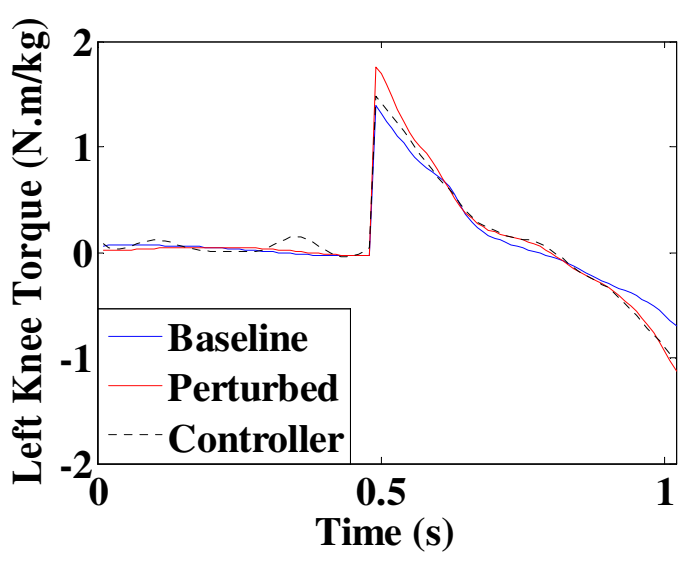

b)

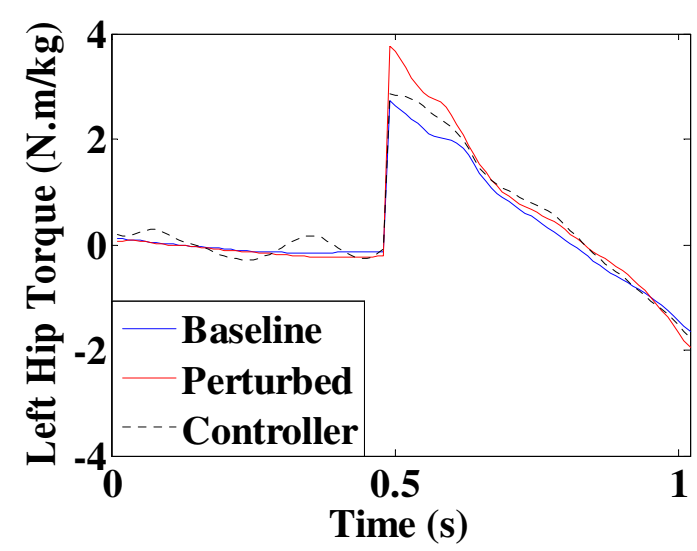

d)

Figure 4.6: Kinetic data for the knee (a), (b) and hip joints (c), (d). Normal (blue) and perturbed (red) data are shown. Predicted applied torque (dashed) is obtained with the best parameters $K_{p i}$ and $K_{d i}$ of the coordinated joint control with time shift. 


\subsubsection{Data Collection}

Ten young (3 females) and three older (male) healthy adults participated in the study. The mean age was $23.2 \pm 2.6$ for young subjects, and $70.67 \pm 5.86$ for older subjects. None of the subjects had prior experience walking with SKB and they were told that the knee axis would lock while they are walking but they were not told which lock strategy would be used or when the lock would engage. Lock strategies were applied in a random order. All subjects gave written informed consent approved by the Institutional Review Board of University of Delaware prior to participating. Safety harness that did not restrict walking and supported no body weight was used during experiments to safeguard subjects from falling. Reflective markers were placed on the subject in order to record joint kinematics via the motion capture system. Ground reaction forces were also measured by an instrumented treadmill (AMTI, Watertown, MA) with independent force platforms under each foot at 1000 Hz. Figure 4.7 shows the experimental setup and a subject wearing the SKB while walking on a treadmill.

The experiments begin by collecting a trial in which subjects walked on the treadmill at a speed of 1 meter/second without wearing the brace. This is followed by an acclimation period of 5 minutes where subjects walk while wearing the brace but no locks engage. At the end of this acclimation period, the baseline pattern of the subject's gait with the brace under the no-lock condition is collected for 1 minute.

After the baseline data are captured, 3 trials of each of 3 locking strategies were used as follows: (i) flexion lock during early-swing, (ii) extension lock in lateswing and (iii) extension lock in mid-stance. Lock trials were run in a random order and each condition was repeated 3 times. Finally, a continuous lock trial is made in which the subject walks for five minutes while the flexion lock is activated in every stride. Prior and subsequent to this trial, the subject is allowed to walk with 


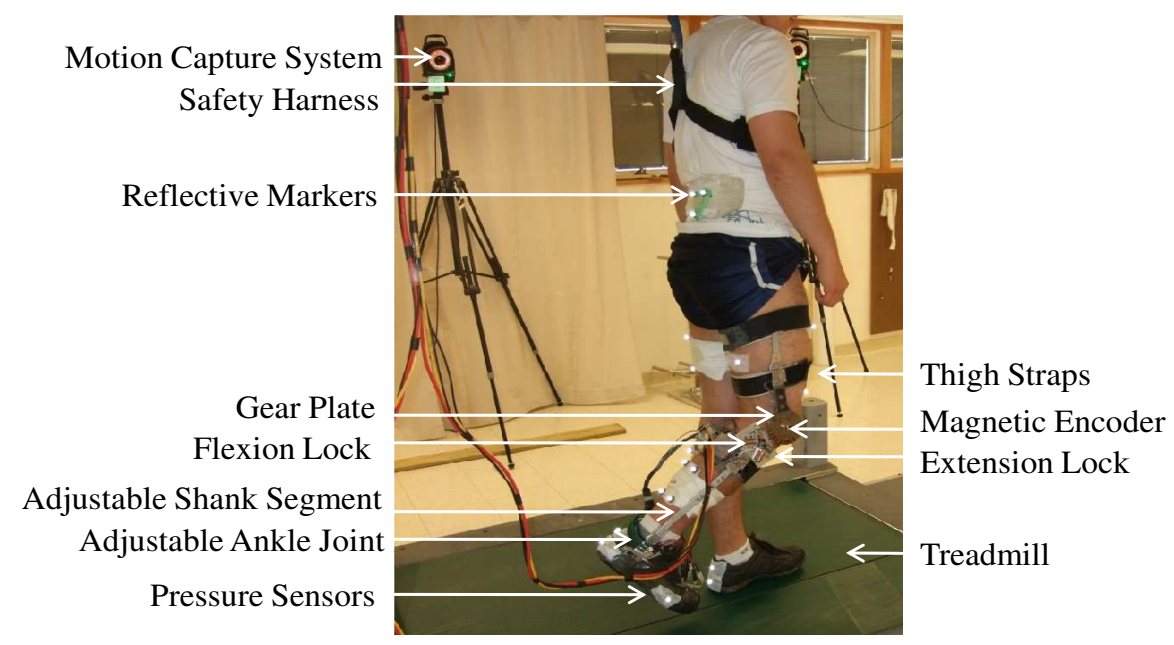

Figure 4.7: Experimental setup - A subject wears SKB on the right leg while walking on a treadmill. A safety harness is used during experiments to protect subjects from falling and avoid possible injuries.

the brace for two minutes without any perturbations. The experimental protocol is summarized in Table 4.1.

\subsubsection{Discussion}

While data have been collected by locking the knee in different phases of the gait cycle, the flexion lock in early swing phase is discussed in this chapter. The parameters calculated by the Coordinated Joint Control with Time Shift after the engagement of the lock on one occasion are described in detail in the following section. One of the key observations from the experiments with healthy adults is as follows: Subjects tend to get back to their normal baseline walking pattern within one stride following the disengagement of the lock in terms of both joint kinematics and kinetics. Figure 4.8 presents Kp vs Kd distribution for younger and older adults computed using the coordinated joint control model with time shift by isolating data from the first stride after the lock is disengaged. 
Table 4.1: EXPERIMENTAL PROTOCOL

\begin{tabular}{cclcc}
\hline Test Bout & $\begin{array}{c}\text { Time } \\
(\text { min })\end{array}$ & $\begin{array}{l}\text { Extension } \\
\text { Lock }\end{array}$ & $\begin{array}{c}\text { Flexion } \\
\text { Lock }\end{array}$ & Gait Cycle \\
\hline Acclimation & 5 & off & off & none \\
Baseline & 1 & off & off & none \\
Test 1 & 2 & off & once & Early Swing \\
Test 2 & 2 & once & off & Mid Stance \\
Test 3 & 2 & once & off & Late Swing \\
Test 4 & 2 & once & off & Mid Stance \\
Test 5 & 2 & once & off & Mid Stance \\
Test 6 & 2 & once & off & Late Swing \\
Test 7 & 2 & off & once & Early Swing \\
Test 8 & 2 & off & once & Early Swing \\
Test 9 & 2 & once & off & Late Swing \\
Test 10-a & 2 & off & off & none \\
Test 10-b & 5 & off & continuous & Early Swing \\
Test 10-c & 2 & off & off & none \\
\hline
\end{tabular}

As seen in Figure 4.8 (e) and (f), the distributions of Kp (position constant) and Kd (velocity constant) parameters show a more scattered response for older subjects. $\mathrm{Kp}$ is a measure of the effect of the perturbation on joint angle while $\mathrm{Kd}$ represents the same effect on the joint velocity. Parameteres from all of the four joints are shown in the figure. Therefore, the scattered response of $\mathrm{Kp}$ and $\mathrm{Kd}$ parameters for older subjects suggests that the engagement of the lock has a more perturbing effect on the gait of older subjects.

As seen in Figure 4.9, optimal parameters in the figure are clustered for different subjects dependent on their neuro-muscular response characteristics. In young adults the response relates more to shortening of the gait cycle duration whereas in older adults, the response to the perturbation related to shorter cycle duration and errors in joint velocity and position. The effect of the perturbation may be used to better understand factors that characterize subjects who are more 
prone to falls under perturbations of the gait. 


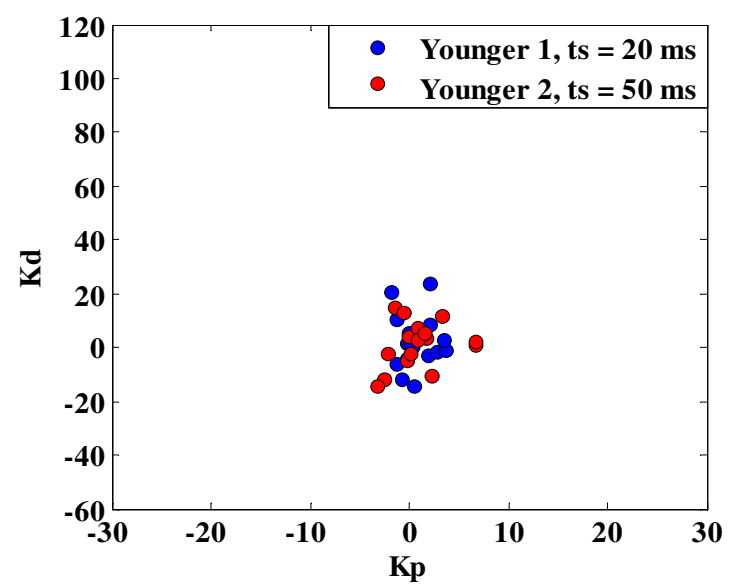

a)

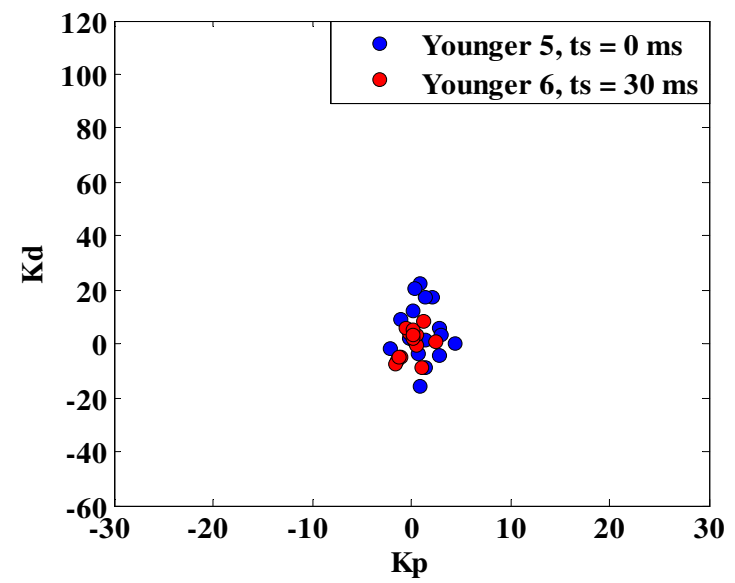

c)

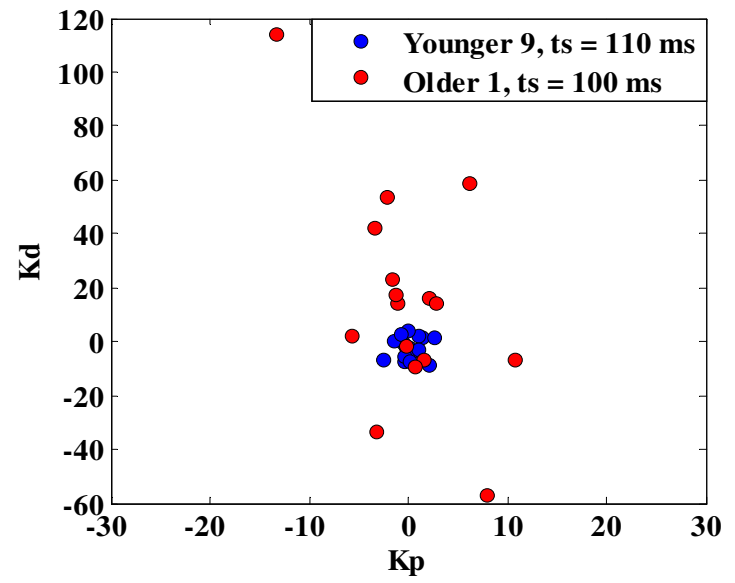

e)

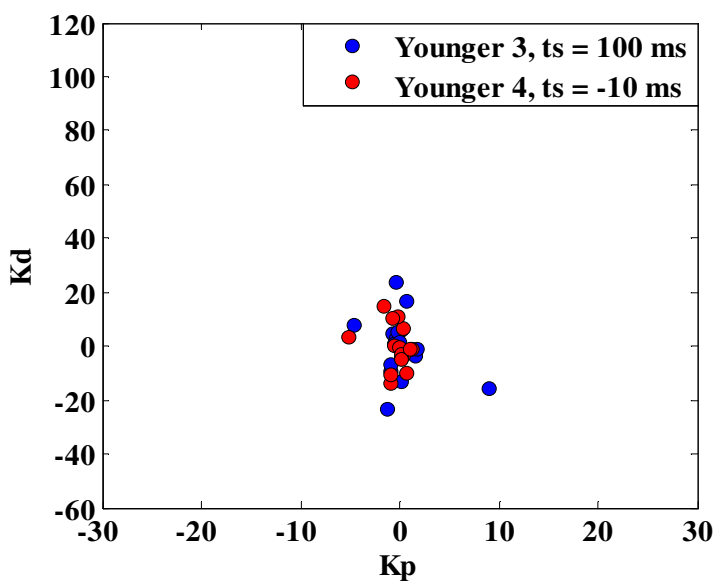

b)

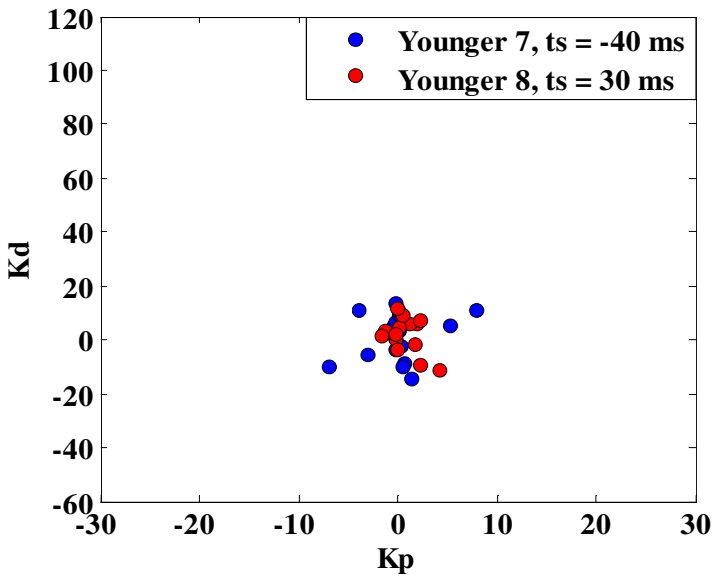

d)

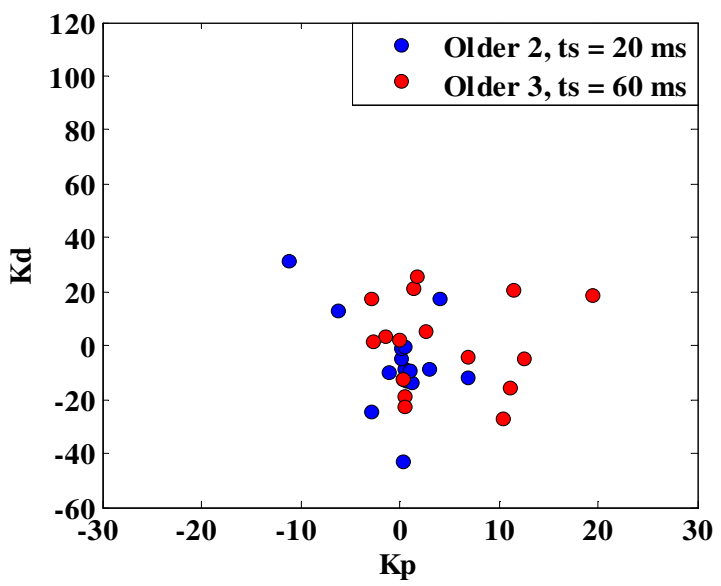

f)

Figure 4.8: Controller parameters Kp (position constant) and Kd (velocity constant) for younger and older subjects. Scatter of the parameters indicate the susceptibility of the subject to the perturbations of the gait. 


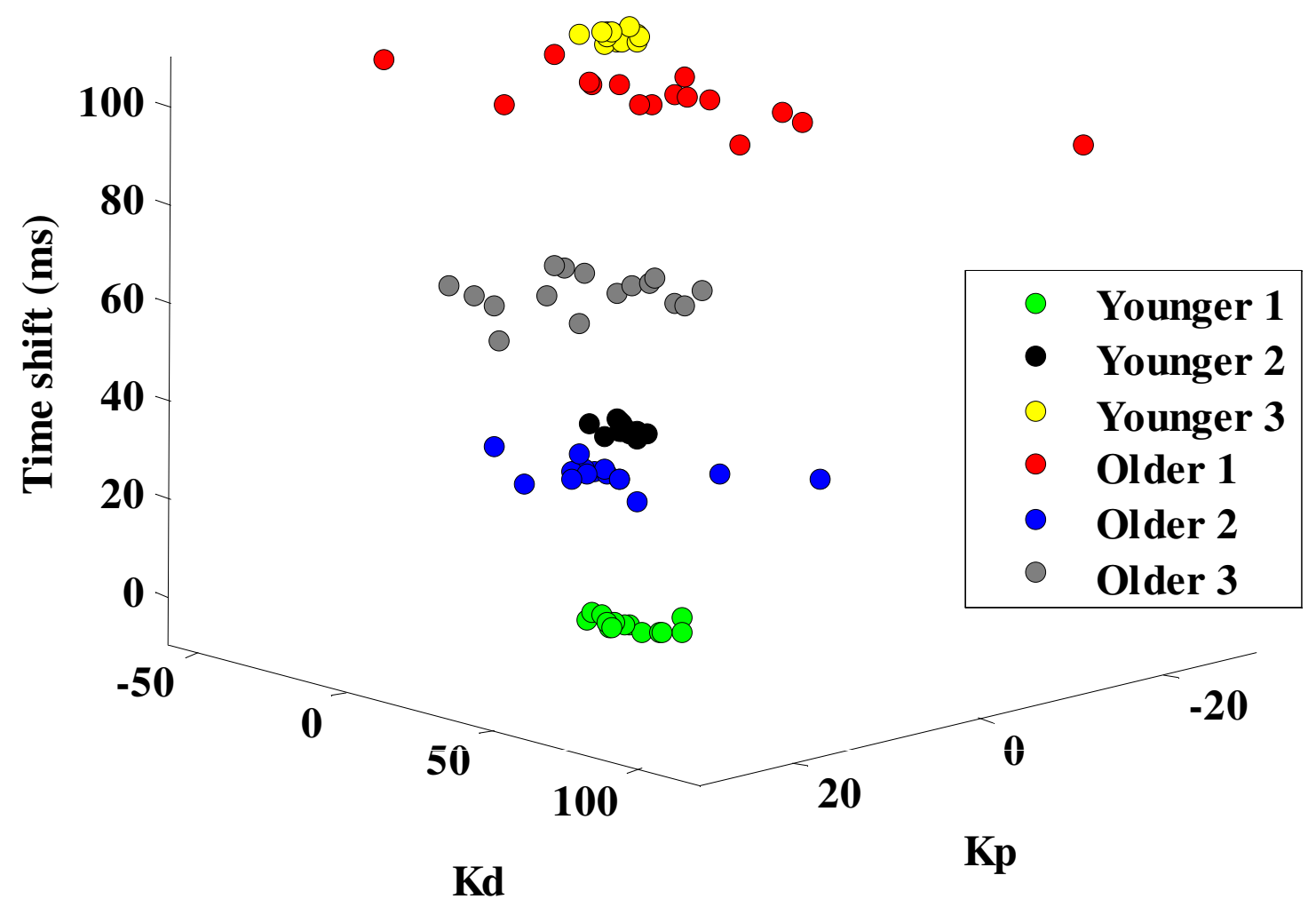

Figure 4.9: Controller parameters of three younger and three older healthy subjects involving the first stride after the disengagement of the lock. Kp, $\mathrm{Kd}$ and time shift values may be used to characterize subjects who are more prone to falling under gait perturbations. 


\section{Chapter 5}

\section{KINEMATICAL GAIT ANALYSIS}

The kinematic responses of subjects to the controlled perturbations are analyzed in order to investigate the effectiveness of the SKB as a gait assistive device. While data have been collected by locking the knee in different phases of the gait cycle, the effects of flexion lock in early swing phase is discussed in this chapter. The response of the subjects to single and sustained gait perturbations are described in detail in the following sections.

\section{$5.1 \quad$ Single Lock}

The effect of flexion lock on peak joint angles is illustrated in Figure 5.1. The lock gets engaged following the toe-off of the right leg and it is kept locked until the subject starts extending the knee.

The first data bar in the figure indicates the average of 10 strides before locking and it is considered as the baseline peak joint angle of the subjects. The graph summarizes the responses of 12 subjects (9 younger and 3 older) across 3 trails. The lock angle is selected as $50^{\circ}$ of flexion as this point in the gait cycle corresponds to the early swing phase of the subjects. Furthermore, the subjects experience a major perturbation to the gait when the knee joint is locked at $50^{\circ}$ due to the following reasons: (i) the subject is in the single support phase of the gait cycle, (ii) the lock is unexpected and occurs much before the subject reaches the peak flexion angle $\left(70^{\circ}\right)$. As the lock angle approaches the peak flexion angle, the effect of the perturbation decreases. As Figure 5.1 illustrates, locking the right knee 


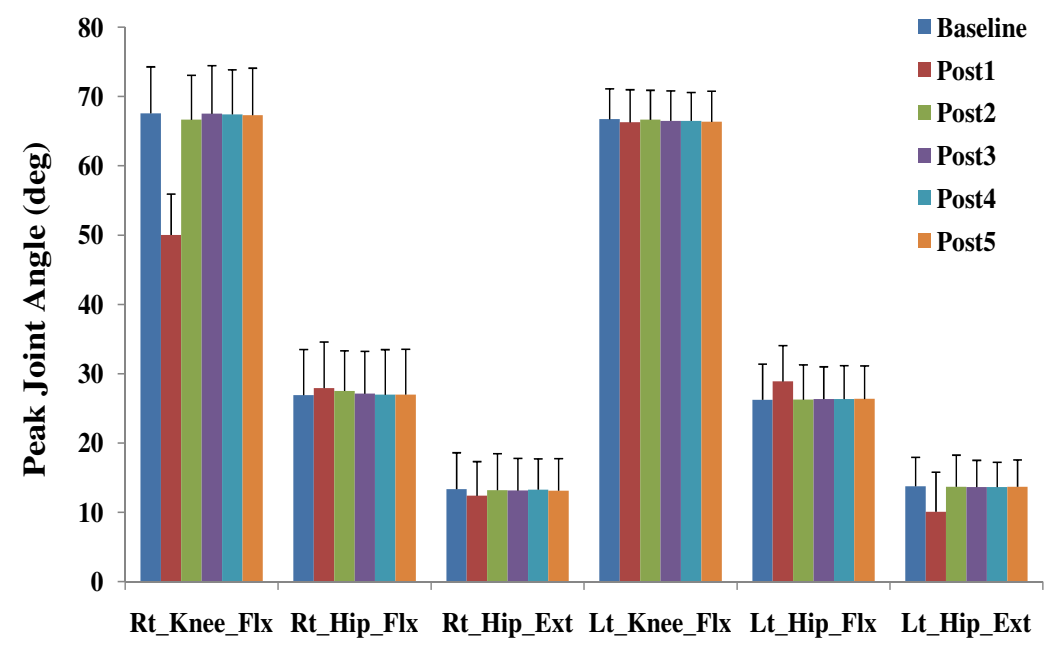

Figure 5.1: Peak joint angles before and after the engagement of the flexion lock in early swing phase. Lock is engaged on one occasion. First data bar indicates the average of 10 strides before locking. Engagement of the lock reduces knee flexion and hip extension while increases hip flexion but the subjects retain their baseline within one stride after the disengagement of the lock.

joint has similar effects on the ipsilateral and contralateral side. In other words, peak knee flexion and hip extension angles are observed to decrease while peak hip flexion angle increases for both legs. It is interesting to note that restricting the right knee flexion during the early swing phase on one occasion has more significant effects on the contralateral hip angles $(p<0.05)$. The effect of the lock on step length is depicted in Figure 5.2.

Engagement of the flexion lock in the early swing phase causes subjects to take a shorter step with the right leg while they take a longer step with the left leg after the lock disengages. Although there is a slight increase in the right hip flexion angle, the right step length deceases mainly due to the decline in the left hip extension. On the contrary, left step length is observed to increase owing to the increase in the left hip flexion angle. Even though the right hip extension decreases 


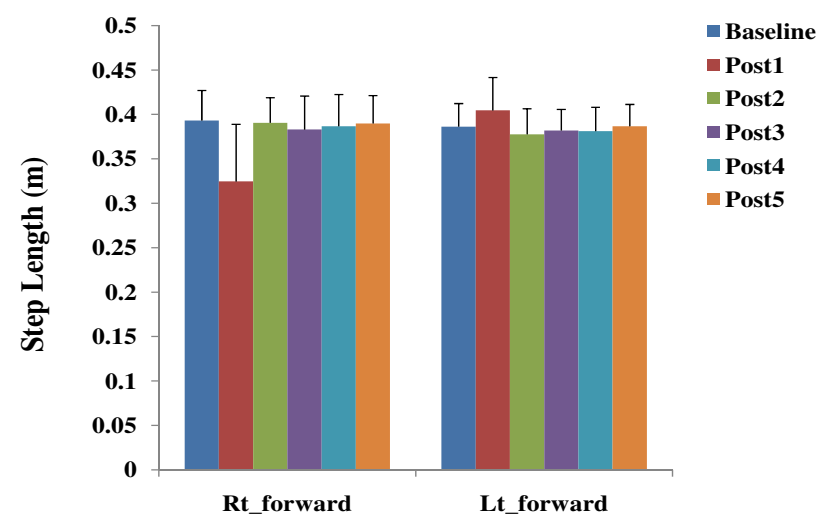

Figure 5.2: Step length is shown for the right leg and the left leg for the average of 10 strides before the engagement of the lock and 5 strides following the disengagement of the lock. Subjects tend to take a shorter step with the right leg while they take a longer step with the left leg due to the perturbation applied to the right knee joint.

after the lock disengages, the left step length increases as the increase in the left hip flexion is higher than the decline in the right hip extension. The effect of the lock on stride durations is shown in Figure 5.3.

Reduced knee flexion in early swing decreases left swing, left double support and right double support durations. It is noteworthy that the right swing time is observed to increase even though the knee joint is locked before the subject reaches the peak knee flexion angle. Moreover, the subjects take a shorter step with the right leg due to the engagement of the lock. Nevertheless, the increase in the right swing time can be explained by the following reasons: (i) the subjects tend to lift up their hips in order to clear their foot from the surface of the treadmill. The circumduction of the right leg causes subjects to spend more time during right swing. (ii) The knee joint is kept locked for a specified amount of time. The lock duration can be set through the software and it is selected as $200 \mathrm{~ms}$ during the experiments; however, 


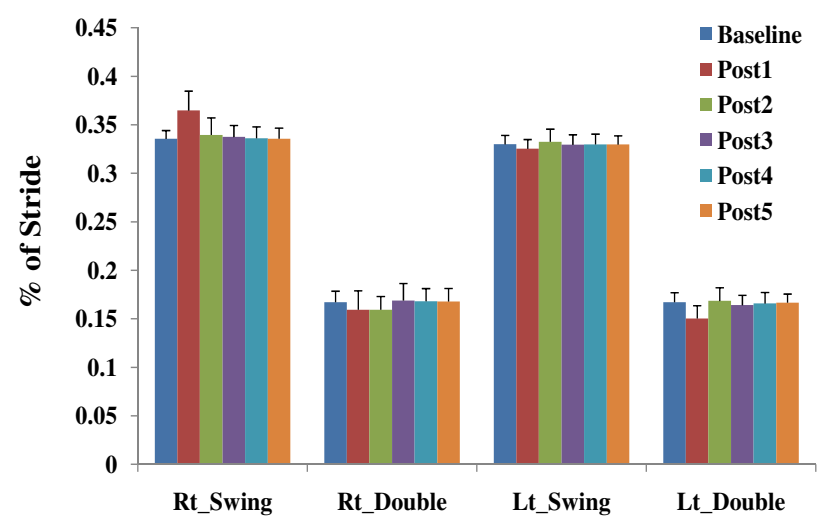

Figure 5.3: Normalized stride durations for the average of 10 strides before the engagement of the lock and 5 strides following the disengagement of the lock. Left swing, left double support and right double support durations decrease while right swing duration increases with the engagement of the lock.

the lock disengages as soon as the subjects realize that they cannot flex any further and start extending the knee. As a result, the actual lock duration depends on the response of the subject and is usually shorter than $200 \mathrm{~ms}$. (iii) In spite of the decrease in the right step length, the right hip flexion increases. Therefore, the right foot lands at a further point on the treadmill compared to the baseline walking.

As soon as the lock is disengaged, healthy adults' gait pattern immediately returns to the pre-lock baseline, thus no adaptation appears to have occurred. Restricting the knee joint motion in early swing phase simulates a variety of activities of daily living where falls occur. This type of perturbation may be useful in balance training to improve righting reactions in people with balance deficits. We believe that exposing subjects to controlled perturbations in gait can be an experience that they can learn; therefore they can recover their balance safely after experiencing such perturbations during the activities of daily living. 


\subsection{Continuous Lock}

During the continuous lock trial, the subject walks for five minutes while the flexion lock is activated in every stride. The effect of the continuous flexion lock on peak joint angles is shown in Figure 5.4 and 5.5.

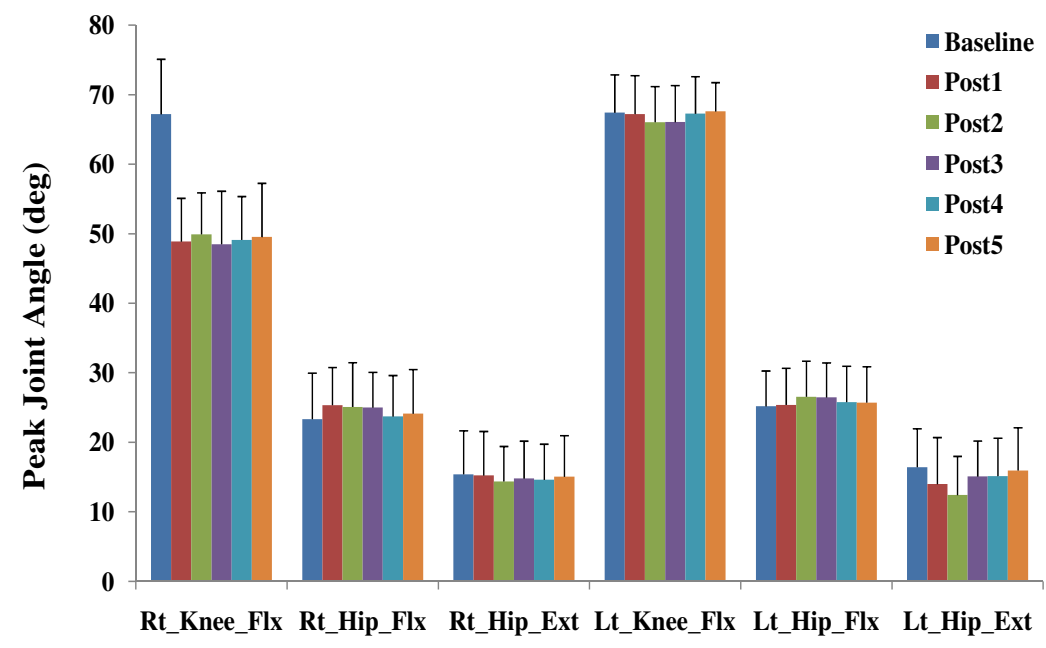

Figure 5.4: Peak joint angles before and after the engagement of the flexion lock in early swing phase. Lock is engaged at every stride for five minutes. First data bar indicates the average of 10 strides before locking. Engagement of the lock reduces knee flexion and hip extension while increases hip flexion.

The first data bar in Figure 5.4 indicates the average of 10 strides before locking and it is considered as the baseline peak joint angle of the subjects. The first five strides of the continuous lock trial are shown in the figure. The graph summarizes the response of 7 subjects ( 5 younger and 2 older) across 3 trails. The lock angle is selected as $50^{\circ}$ of flexion due to the reasons mentioned in the previous section. The effects of the continuous lock on peak joint angles are similar to the effects observed during the single lock trial. Consequently, peak knee flexion and hip extension angles are observed to decrease while peak hip flexion angle increases for both legs during the first five strides of continuous locking. 


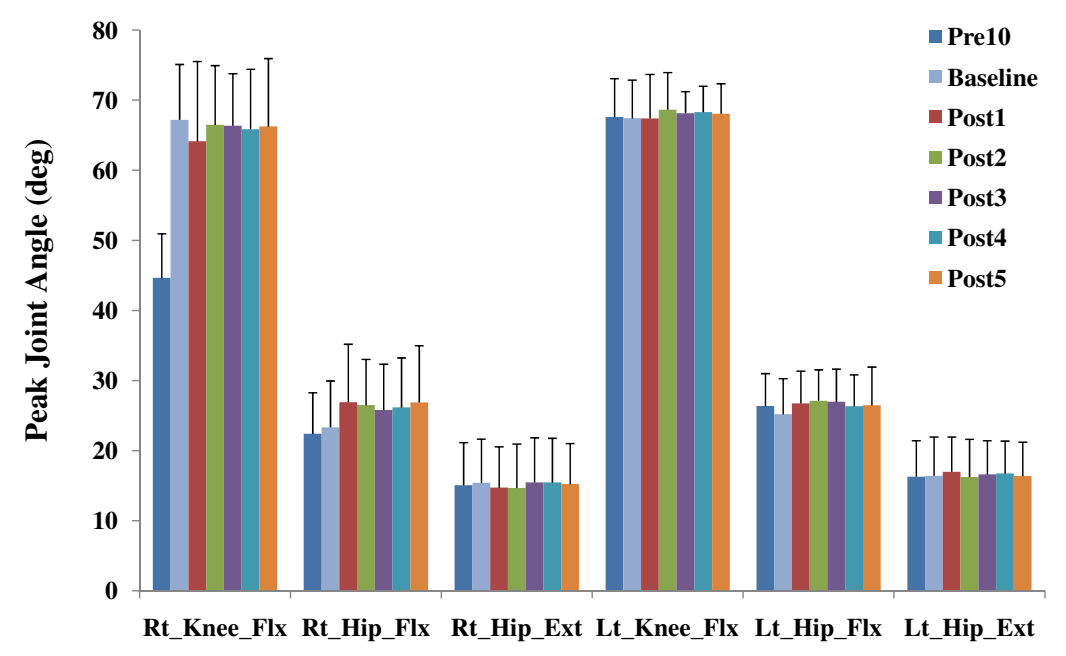

Figure 5.5: Peak joint angles before and after the disengagement of the flexion lock in early swing phase. First data bar indicates the average of 10 strides before the disengagement of the lock. Second data bar shows the baseline peak joint angles for pre-locking condition. Hip flexion increases significantly following the release of the lock.

The lock engagement stops at the end of five minutes. Peak right knee flexion angle following the disengagement of the lock is less than the baseline peak value because the subject is not prepared for the cessation of lock engagement, as shown in Figure 5.5. Upon removal of the resistance, subjects exhibit aftereffects on peak joint angles. It is important to note that the increase in right hip and left hip flexion angles are more significant than other joint angles. The effect of the continuous lock on step length is depicted in Figure 5.6 and 5.7.

The step length pattern is similar to the one observed during single lock trials as shown in Figure 5.6. Engagement of the flexion lock in the early swing phase causes subjects to take a shorter step with the right leg while they take a longer step with the left leg when compared to the baseline walking pattern. On the other hand, subjects tend to recover their baseline step lengths during the first five strides as they realize the continuity of locking. 


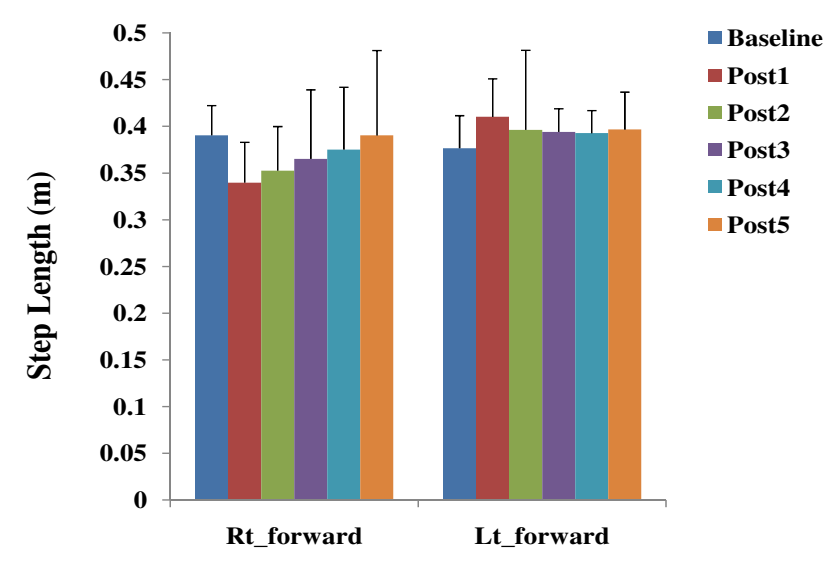

Figure 5.6: Step length is shown for the right leg and the left leg for the average of 10 strides before the engagement of the lock and the first 5 strides of continuous locking. Subjects tend to take a shorter step with the right leg while they take a longer step with the left leg due to the perturbation applied to the right knee joint.

The first data bar in Figure 5.7 indicates the average of 10 strides before the disengagement of the lock and the second data bar shows the baseline step length for pre-locking condition. As far as the first two data bars are concerned, subjects adapt a shorter right step length and a longer left step length at the end of five minutes. An overshoot in both right and left step lengths is observed with the disengagement of lock at the end of continuous locking and this increase in step length is mainly attributed to the increase in right hip and left hip flexion angles. The effect of the continuous lock on stride durations is depicted in Figure 5.8 and 5.9.

As observed in the single lock strategy, reduced knee flexion in early swing increases the right swing duration significantly. As the subjects realize the continuity of locking, the hiking of the hip reduces, which explains the decreasing pattern in the right swing duration during the first five strides of locking. Also, double support durations decrease for both legs. As opposed to the single lock trial, an increasing 


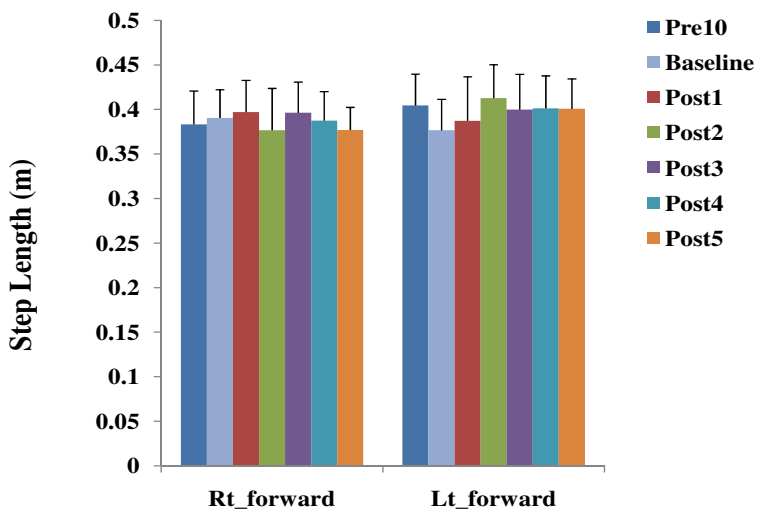

Figure 5.7: Step length before and after the disengagement of the flexion lock in early swing phase. First data bar indicates the average of 10 strides before the disengagement of the lock. Second data bar shows the baseline step length for pre-locking condition. An overshoot in both right and left step lengths is observed with the disengagement of lock at the end of five minutes.

pattern in left swing duration is observed during the first five strides of continuous locking.

The first data bar in Figure 5.9 indicates the average of 10 strides before the disengagement of the lock and the second data bar shows the baseline duration for pre-locking condition. Subjects adapt a longer right swing duration while left swing, left double support and right double support durations decrease at the end of five minutes. An overshoot in right swing duration is observed with the disengagement of the lock and subjects adapt longer right swing duration at the end of continuous locking.

The repeated restriction in knee flexion during swing induced adaptation is indicated by an aftereffect of greater hip flexion in the strides following knee release. Training under new conditions implies the formation or recalibration of the motor 


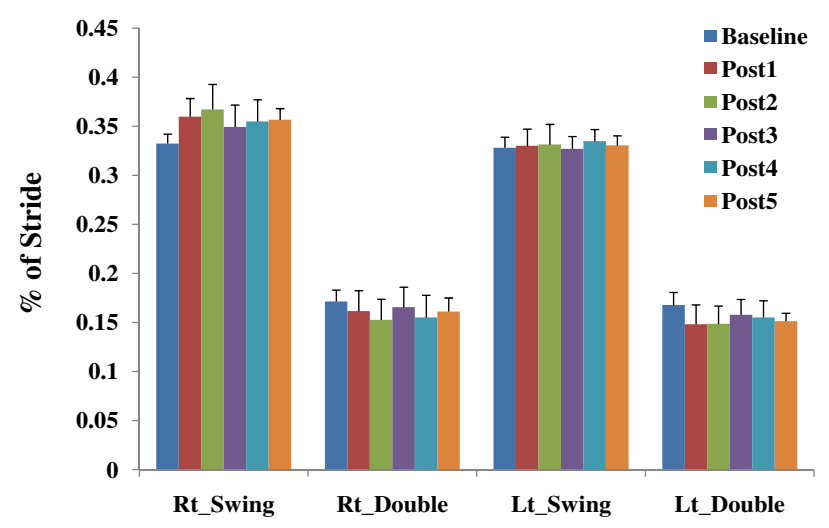

Figure 5.8: Normalized stride durations for the average of 10 strides before the engagement of the lock and the first 5 strides of continuous locking. Double support durations decrease while swing durations increase with the engagement of the lock.

output for a given task [13]. The persistently lower knee flexion after the lock is released indicates that this strategy might be useful in inducing longer term changes in kinematic patterns, which may be useful in gait retraining activities. The CNS must be able to accommodate the changes in the mechanical properties of our limbs to ensure safe locomotion [14]. As seen in Fig. 5.7, subjects tend to take longer steps after repeated restriction in the knee flexion. Studies with stroke subjects show that constraint induced movement therapy resulted in improvements in the amount of use of the affected upper extremity $[36,37]$. We believe that immobilizing the stronger side forces subjects to learn to use the other side. The swing induced therapy is indicated by an increase in left stance time, peak hip flexion and step length due to the increased joint range of motion in the contralateral side. The SKB may be an effective rehabilitation device for stroke subjects. 


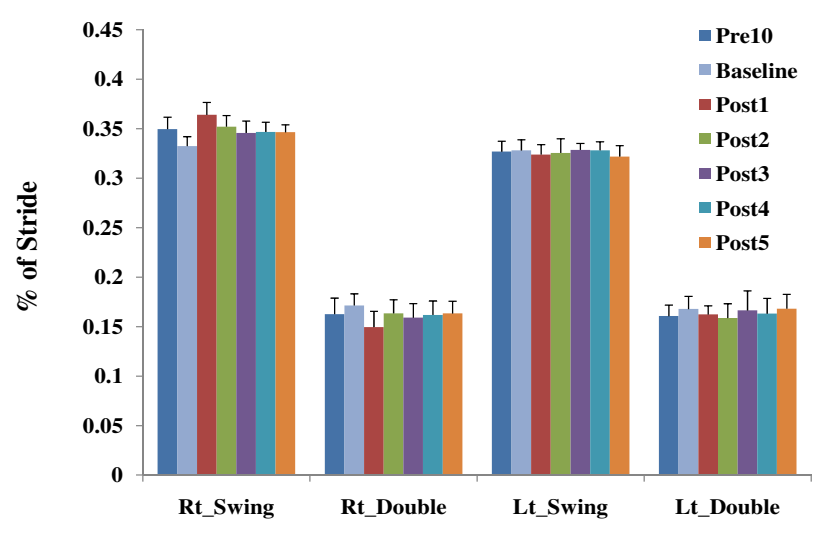

Figure 5.9: Normalized stride durations before and after the disengagement of the flexion lock in early swing phase. First data bar indicates the average of 10 strides before the disengagement of the lock. Second data bar shows the baseline duration for pre-locking condition. Subjects adapt a longer right swing duration while left swing, left double support and right double support durations decrease at the end of five minutes. 


\section{Chapter 6}

\section{CONCLUSIONS}

The SKB is used to develop a new method to categorize subjects that are prone to falls and to induce righting reactions and longer term changes in kinematic patterns that might be useful in preventing falls. The SKB allows physical therapist the ability to research, develop and implement effective training practices for gait rehabilitation through motion learning and feedback to the human neuromuscular system. Improvements on the previous design has led to the current effective gait training device. Previous shorthcomings dealing with the fit of the brace and control algorithm have been resolved. The use of padding and adjustable ankle segment help to maintain proper alignment between the knee and ankle joints of the human with the knee and ankle joints on the brace. Thus, the leg has been prevented from moving inside the brace while allowing the subject to walk comfortably with the device. Experiments have been run with younger and older subjects owing to the versality of the SKB, which allows for a broader subject pool.

The shortcomings of the SKB reside in the lock mechanism. Even though the gear plate allows locking the knee joint at various angles during different phases of the gait cycle, engaging the locks at certain points of the gait cycle are not feasible due to the current design. Also, instead of using padding, thigh and shank segments on the brace must be replaced with adjustable segments, which would take care of the customized frame design and allow a much broader subject pool. 


\subsection{Future Work}

The engagement of the extension lock during mid-swing phase cannot be achieved due to the design of the gear plate. However, restricting the knee joint motion in mid-swing phase simulates a variety of activities of daily living where falls occur due to tripping. Therefore, being able to apply controlled perturbations during this phase of the gait cycle has a crucial effect in inducing righting reactions that might be useful in preventing falls. For this reason, the gear plate needs to be redesigned as shown in Figure 6.1.

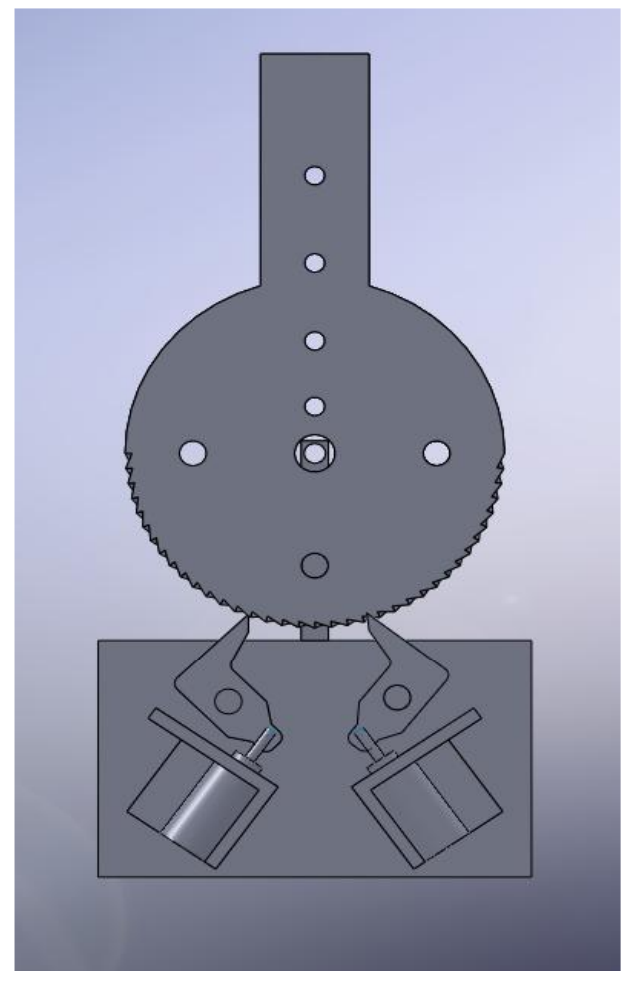

a)

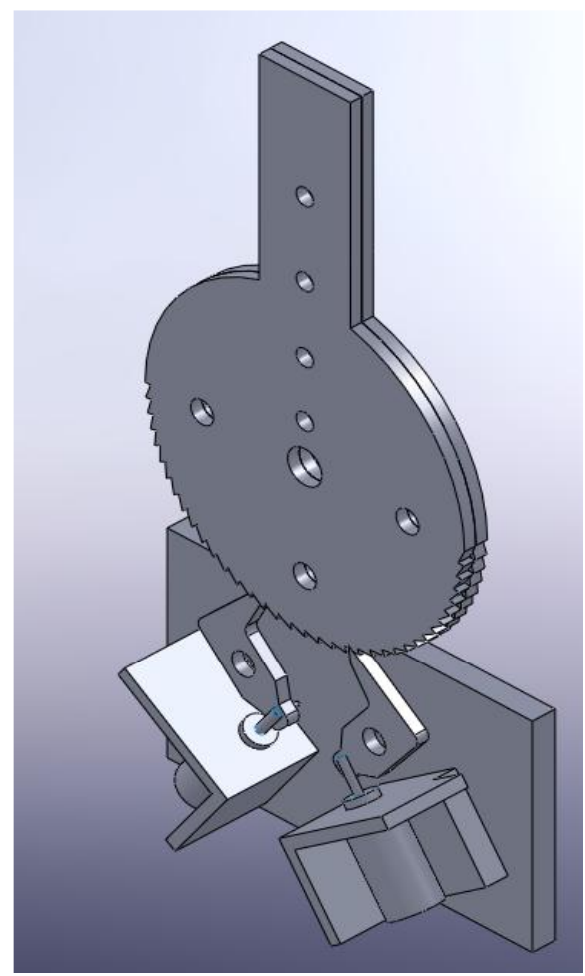

b)

Figure 6.1: Lock mechanism with separate extension and flexion gear plates.

The new design involves two separate gear plates on top of each other. One of the plates is designed for locking the knee joint during flexion while the other one 
locks the joint during extension. Therefore, the knee joint can effectively be locked at any angle during different phases of the gait cycle. Engagement of the locks at any point in the gait cycle and replacing thigh and shank segments with new parts that enable to adjust the segment width would make the SKB an extremely powerful tool in gait rehabilitation. 


\section{BIBLIOGRAPHY}

[1] J. P. White, Real time event detection and control of a smart knee brace for gait rehabilitation, MS Thesis, University of Delaware, 2007.

[2] W. E. Prentice, M. L. Voight, Techniques in musculoskeletal rehabilitation, 1st ed. USA: McGraw-Hill Professional, 2001.

[3] D. A. Winter, Biomechanics and motor control of human movement, 3rd ed. Hoboken, New Jersey: John Wiley \& Sons, Inc., 2005.

[4] S. R. Lord, C. Sherrington, H. B. Menz, Falls in older people: risk factors and strategies for prevention, 1st ed. Cambridge, United Kingdom: Cambridge University Press, 2001.

[5] R. Tideiksaar, Falls in older people: prevention and management, 3rd ed. Baltimore, Maryland: Health Professions Press, Inc., 2002.

[6] WHO global report on falls prevention in older age, France: World Health Organization, 2008.

[7] J. H. van Dieën, M. Pijnappels, M. F. Bobbert, "Age-related intrinsic limitations in preventing a trip and regaining balance after a trip," Eur J Appl Physiol, vol. 43, no. 7, pp. 437-453, 2005.

[8] R. Ferber, L. R. Osternig, M. H. Woollacott, N. J. Wasielewski, J. H. Li, "Reactive balance adjustments to unexpected perturbations during human walking," Gait Posture, vol. 16, no. 3, pp. 238-248, 2002.

[9] H. J. A. van Hedel, M. Biedermann, T. Erni, V. Dietz, "Obstacle avoidance during human walking: transfer of motor skill from one leg to the other," $J$ Physiol, vol. 543, no. 2, pp. 709-717, 2002.

[10] J. L. Emken, J. H. Wynne, S. J. Harkema, D. J. Reinkensmeyer, "A robotic device for manipulating human stepping," IEEE Trans Robot, vol. 22, no. 1, pp. 185-189, 2006.

[11] K. E. Gordon, D. P. Ferris, "Learning to walk with a robotic ankle exoskeleton," J Biomech, vol. 40, no. 12, pp. 2636-2644, 2007. 
[12] S. Hesse, D. Uhlenbrock, "A mechanized gait trainer for restoration of gait," $J$ Rehabil Res Dev, vol. 37, no. 6, pp. 701-708, 2000.

[13] T. Lam, M. Anderschitz, V. Dietz, "Contribution of feedback and feedforward strategies to locomotor adaptations," J Neurophysiol, vol. 95, no. 2, pp. 766$773,2006$.

[14] J. W. Noble, S. D. Prentice, "Adaptation to unilateral change in lower limb mechanical properties during human walking," Exp Brain Res, vol. 169, no. 4, pp. 482-495, 2006.

[15] A. Mansfield, A. L. Peters, B. A. Liu, B. E. Maki, "A perturbations-based balance training program for older adults: study protocol for a randomised controlled trial," BMC Geriatr, vol. 7, no. 12, pp. 1-14, 2007.

[16] D. S. Marigold, A. E. Patla, "Adapting locomotion to different surface compliances: neuromuscular responses and changes in movement dynamics," J Neurophysiol, vol. 94, no. 3, pp. 1733-1750, 2005.

[17] T. Masud, R. O. Morris, "Epidemiology of falls," Age Aging, vol. 30, suppl. 4, pp. 3-7, 2001.

[18] L. I. E. Oddsson, C. Wall, M. D. McPartland, D. E. Krebs, C. A. Tucker, "Recovery from perturbations during paced walking," Gait Posture, vol. 19, no. 1, pp. 24-34, 2003.

[19] A. E. Patla, S. D. Prentice, C. Robinson, J. Neufeld, "Visual control of locomotion: strategies for changing direction and for going over obstacles," J Exp Psychol Hum Percep Perform, vol. 17, no. 3, pp. 603-634, 1991.

[20] M. J. Pavol, T. M. Owings, K. T. Foley, M. D. Grabiner, "Mechanisms leading to a fall from an induced trip in healthy older adults," J Gerontol Med Sci, vol. 56A, no. 7, pp. M428-M437, 2001.

[21] M. J. Pavol, E. F. Runtz, B. J. Edwards, Y. C. Pai, "Age influences the outcome of alipping perturbation during initial but not repeated exposures," J Gerontol Med Sci, vol. 57A, no. 8, pp. M496-M503, 2002.

[22] M. Pijnappels, J. C. E. van der Burg, N. D. Reeves, J. H. van Dieën, "Identifying of elderly fallers by muscle strength measures," Eur J Appl Physiol, vol. 72, no. 1, pp. 81-88, 2005.

[23] M. Pijnappels, M. F. Bobbert, J. H. van Dieën, "Push-off reactions in recovery after tripping discriminate young subjects, older non-fallers and older fallers," Gait Posture, vol. 21, no. 4, pp. 388-394, 2005. 
[24] S. S. Rao, "Prevention of falls in older patients," Am Fam Physician, vol. 102, no. 5, pp. 585-592, 2008.

[25] B. S. Roudsari, B. E. Ebel, P. S. Corso, N. A M. Molinari, T. D. Koepsell, "The acute medical care costs of fatal-related injuries among the U.S. older adults," Injury, vol. 36, no. 11, pp. 1316-1322, 2005.

[26] A. M. Schillings, B. M. H. Van Wezel, J. Duysens, "Mechanically induced stumbling during human treadmill walking," J Neurosci Methods, vol. 67, no. 1, pp. 11-17, 1996.

[27] D. A. Skelton, J. Kennedy, O. M. Rutherford, "Explosive power and asymmetry in leg muscle function in frequent fallers and non-fallers aged over 65," Age Aging, vol. 31, no. 2, pp. 119-125, 2005.

[28] V. S. Stel, J. H. Smit, S. M. F. Pluijm, P. Lips, "Balance and mobility performance as treatable risk factors for recurrent falling in older persons," J Clin Epidemiol, vol. 56, no. 7, pp. 659-668, 2003.

[29] J. A. Stevens, P. S. Corso, E. A. Finkelstein, T. R. Miller, "The costs of fatal and non-fatal falls among older adults," Inj Prev, vol. 12, no. 5, pp. 290-295, 2006.

[30] P. F. Tang, M. H. Woollacott, R. K. Y. Chong, "Control of reactive balance adjustments in pertrubed human walking: roles of proximal and distal postural muscle activity," Exp Brain Res, vol. 119, no. 2, pp. 141-152, 1998.

[31] D. A. Winter, "Human balance and posture control during standing and walking," Gait Posture, vol. 3, no. 4, pp. 193-214, 1995.

[32] E. T. Wolbrecht, V. Chan, D. J. Reinkensmeyer, J. E. Bobrow, "Optimizing compliant, model-based robotic assistance to promote neurorehabilitation," IEEE Trans Neural Syst Rehabil Eng, vol. 16, no. 3, pp. 286-296, 2008.

[33] M. H. Woollacott, P. F. Tang, "Balance control during walking in the older adults: research and its implications," Phys Ther, vol. 77, no. 6, pp. 646-660, 1997.

[34] W. Yu, Y. Ikemoto, "An artificial reflex improves the perturbation-resistance of a human walking simulator," Med Bio Eng Comput, vol. 45, no. 11, pp. 1095-1104, 2007.

[35] E. P. Zehr, T. Komiyama, R. B. Stein, "Cutaneous reflexes during human gait: electromyographic and kinematic responses to electrical stimulation," $J$ Neurophysiol, vol. 77, no. 11, pp. 3311-3325, 1997. 
[36] S. L. Wolf, C. J. Winstein, J. P. Miller, E. Taub, G. Uswatte, D. Morris, C. Giuliani, K. E. Light, D. N. Larsen, "Effect of constraint-induced movement therapy on upper extremity function 3 to 9 months after stroke," JAMA, vol. 296, no. 17 , pp. 2095-2104, 2006.

[37] W. H. R. Miltner, H. Bauder, M. Sommer, C. Dettmers, E. Taub, "Effects of constraint-induced movement therapy on patients with chronic motor deficits after stroke," Stroke, vol. 30, pp. 586-592, 1999. 